\title{
A MULTISHIFT, MULTIPOLE RATIONAL QZ METHOD WITH AGGRESSIVE EARLY DEFLATION*
}

\author{
DAAN CAMPS $^{\dagger}, \mathrm{KARL}_{\mathrm{MEERBERGEN}}^{\dagger}, \mathrm{AND}^{\mathrm{RAF}} \mathrm{VANDEBRIL}^{\dagger}$
}

\begin{abstract}
The rational QZ method generalizes the QZ method by implicitly supporting rational subspace iteration. In this paper we extend the rational QZ method by introducing shifts and poles of higher multiplicity in the Hessenberg pencil. The result is a multishift, multipole iteration on block Hessenberg pencils. In combination with tightly-packed shifts and advanced deflation techniques such as aggressive early deflation we obtain an efficient method for the dense generalized eigenvalue problem. Numerical experiments demonstrate the level 3 BLAS performance and compare the results with LAPACK routines for the generalized eigenvalue problem. We show that our methods can outperform all in terms of speed and accuracy and observe an empirical time complexity significantly lower than $O\left(n^{3}\right)$.
\end{abstract}

Key words. generalized eigenvalues, rational QZ, rational Krylov, multishift, multipole, aggressive early deflation, software

AMS subject classifications. $65 \mathrm{~F} 15,15 \mathrm{~A} 18$

1. Introduction. The rational QZ method (RQZ) [6] generalizes the standard QZ method of Moler \& Stewart [16]. Both are methods for the numerical solution of the dense, unsymmetric generalized eigenvalue problem defined by a pair of matrices $A, B \in \mathbb{F}^{n \times n}, \mathbb{F} \in\{\mathbb{C}, \mathbb{R}\}$. The set of eigenvalues of the pencil $(A, B)$ is denoted as $\Lambda$ and defined by,

$$
\Lambda=\{\lambda=\alpha / \beta \in \overline{\mathbb{C}}: \operatorname{det}(\beta A-\alpha B)=0\},
$$

with $\overline{\mathbb{C}}=\mathbb{C} \cup\{\infty\}$. Eigenvalues with $\beta=0$ are located at $\infty$. We assume throughout this paper that the pair $(A, B)$ is regular which means that its characteristic polynomial differs from zero. This implies that there are exactly $n$ eigenvalues including these at $\infty$.

The RQZ method acts on pencils in Hessenberg, Hessenberg form instead of the Hessenberg, triangular form used in the QZ method. It relies on pole swapping instead of bulge chasing. Both the single shift RQZ method and the RQZ method with tightly-packed shifts, as formulated in [6], are applicable to real- and complexvalued pencils. However it requires complex arithmetic for real-valued pencils having complex conjugate eigenvalues. The RQZ method computes the generalized Schur form of $(A, B)$. This is a unitary equivalence transformation,

$$
(T, S)=Q^{*}(A, B) Z,
$$

such that $(T, S)$ is a triangular, triangular pencil equivalent to $(A, B)$. The eigenvalues of $(A, B)$ are readily available as the ratios $t_{i i} / s_{i i}$ of the diagonal elements.

In this paper we introduce the multishift, multipole RQZ method which acts on pencils where both matrices are in block Hessenberg form. The main benefit of using shifts and poles of higher multiplicity is that complex conjugate pairs of shifts and

\footnotetext{
* Submitted to the editors February 25, 2019.

Funding: The research was partially supported by the Research Council KU Leuven, projects C14/16/056 (Inverse-free Rational Krylov Methods: Theory and Applications), OT/14/074 (Numerical algorithms for large scale matrices with uncertain coefficients)

${ }^{\dagger}$ Department of Computer Science, KU Leuven, University of Leuven, 3001 Leuven, Belgium. (daan.camps@kuleuven.be, karl.meerbergen@kuleuven.be,raf.vandebril@kuleuven.be)
} 
poles can be represented in real arithmetic for real-valued pencils. This is similar to the well-known implicit double-shift QR step introduced by Francis [9] and the double-shift QZ step [16]. The focus of this paper is thus on the case $\mathbb{F}=\mathbb{R}$. The multishift, multipole RQZ method no longer converges to the triangular, triangular pencil of the generalized Schur form (2). Instead, for $A, B \in \mathbb{R}^{n \times n}$, it will converge to the real generalized Schur form,

$$
(S, T)=Q^{T}(A, B) Z=\left(\left[\begin{array}{cccc}
S_{11} & S_{12} & \ldots & S_{1 m} \\
0 & S_{22} & \ddots & S_{2 m} \\
\vdots & \ddots & \ddots & \vdots \\
0 & \ldots & 0 & S_{m m}
\end{array}\right],\left[\begin{array}{cccc}
T_{11} & T_{12} & \ldots & T_{1 m} \\
0 & T_{22} & \ddots & T_{2 m} \\
\vdots & \ddots & \ddots & \vdots \\
0 & \ldots & 0 & T_{m m}
\end{array}\right]\right)
$$

where the diagonal subpencils $\left(S_{i i}, T_{i i}\right), i=1, \ldots, m$ are of dimension $1 \times 1$ and $2 \times 2$ and correspond with respectively the real and complex conjugate eigenvalues of $(A, B)$.

The remainder of this article consists out of two parts. Sections 2 and 3 make up the theoretical part of the paper. In Section 2, we study matrix pencils in block Hessenberg form. We define properness of block Hessenberg pencils, their pole pencil, and their pole tuple. We show how the pole tuple can be altered by changing pole blocks at the edge of the pencil and by swapping neighboring pole blocks. The multishift, multipole RQZ step follows directly from this discussion. Section 3 extends the implicit Q theorem for Hessenberg pencils [6] to block Hessenberg pencils and briefly discusses the convergence behaviour of the method.

In the second part of the paper, we follow a practical approach and discuss how a multishift, multipole RQZ method can be implemented in finite precision arithmetic. The QR method suffers from a degraded performance when moderate to large shift multiplicities are used. Watkins [18] studied this phenomenon and demonstrated that shifts become blurred during a QR iteration of higher shift multiplicity. This severely decreases the effectiveness of the shifts. For the QR method, this issue is mitigated in the small bulge multishift variant introduced by Braman, Byers \& Mathias [3]. This approach is extended to the QZ method by Kågström \& Kressner [12]. In Section 4, we demonstrate that the multishift, multipole RQZ method is also prone to numerical issues when shifts and poles of moderate to large multiplicities are used. To overcome these numerical difficulties, we propose a multishift, multipole RQZ method that uses tightly-packed, small blocks. Specifically, blocks of dimension $2 \times 2$ for complex conjugate shifts and poles in real pencils and of dimension $1 \times 1$ for real shifts and poles in real pencils and in complex pencils. The last tool we adapt from recent improvements to the QR [4] and QZ [12] methods to the RQZ method is the use of advanced deflation strategies. Specifically we implement the aggressive early deflation technique during the RQZ iteration in order to obtain level 3 BLAS performance.

The resulting methods are implemented as part of the Fortran package libRQZ which is made publicly available at numa.cs.kuleuven.be/software/rqz. Section 6 illustrates the performance of libRQZ with some numerical experiments. We conclude the paper in Section 7.

Notation and elementary definitions. $A, B, \ldots$ are matrices, $\boldsymbol{a}, \boldsymbol{b}, \ldots$ vectors, and $\alpha, \beta, \ldots$ scalars. Subspaces are denoted with calligraphic letters. For example, $\mathcal{R}(A)=\mathcal{R}\left(\boldsymbol{a}_{1}, \ldots, \boldsymbol{a}_{n}\right)$ is the column space of $A=\left[\boldsymbol{a}_{1} \ldots \boldsymbol{a}_{n}\right], \mathcal{E}_{k}=\mathcal{R}\left(\boldsymbol{e}_{1}, \ldots, \boldsymbol{e}_{k}\right)$ with $\boldsymbol{e}_{j}$ the $j$ th canonical basis vector of appropriate dimension. The $k$ th order Krylov subspace generated by $A$ from starting vector $\boldsymbol{v}$ is $\mathcal{K}_{k}(A, \boldsymbol{v})=\mathcal{R}\left(\boldsymbol{v}, A \boldsymbol{v}, \ldots, A^{k-1} \boldsymbol{v}\right)$. The tuple $(\alpha, \beta)$ is ordered and $\{\alpha, \beta\}$ denotes an unordered multiset with repetition. 
The complex plane extended with the point at infinity, $\mathbb{C} \cup\{\infty\}$, is denoted as $\overline{\mathbb{C}}$. Division of a nonzero $\alpha \in \mathbb{C}$ by 0 results in infinity.

2. Block Hessenberg pencils. In the first part of this section we define block Hessenberg matrices and pencils and study their characteristics. The second part of this section recapitulates the main results on rational Krylov theory from [6] to prove that rational Krylov spaces generated from block Hessenberg pencils have a block structure. The third and last part of this section describes two relevant operations on a block Hessenberg pencil.

2.1. Definitions and elementary results. We first define a block upper triangular matrix and the notation we will use for it.

Definition 2.1. A matrix $R \in \mathbb{F}^{n \times n}$ is called a block upper triangular matrix with block partition $s=\left(s_{1}, \ldots, s_{m}\right), s_{1}+\ldots+s_{m}=n$, if it admits the form,

$$
\left[\begin{array}{cccc}
R_{11} & R_{12} & \ldots & R_{1 m} \\
& R_{22} & \ldots & R_{2 m} \\
& & \ddots & \vdots \\
& & & R_{m m}
\end{array}\right],
$$

with block $R_{j k}$ of size $s_{j} \times s_{k}$ for $1 \leq j \leq k \leq m$. The vector $s$ defines the sizes of the blocks and is called the partition vector. For the sake of clarity, the block partition can be explicitly denoted as $R_{\left(s_{1}, \ldots, s_{m}\right)}$ or $R_{\boldsymbol{s}}$.

A special case of a block upper triangular matrix is a block diagonal matrix $D_{s}$ in which all off-diagonal blocks are zero:

$$
D_{\boldsymbol{s}}=\left[\begin{array}{llll}
D_{11} & & & \\
& D_{22} & & \\
& & \ddots & \\
& & & D_{m m}
\end{array}\right] .
$$

We sometimes use the notation $D_{s}=\operatorname{diag}\left(D_{11}, D_{22}, \ldots, D_{m m}\right)$ for block diagonal matrices. Further note that if $R_{s}$ is a nonsingular block upper triangular matrix, $\hat{R}_{s}=R_{s}^{-1}$ is also a block upper triangular matrix with an identical block partition $s$.

Next we define a block upper Hessenberg matrix based on the definition of a block upper triangular matrix.

Definition 2.2. A matrix $H \in \mathbb{F}^{n \times n}$ is called a block upper Hessenberg matrix with block partition $s=\left(s_{1}, \ldots, s_{m}\right), s_{1}+\ldots+s_{m}=n-1$, if it admits the form,

$$
H_{\boldsymbol{s}}=\left[\begin{array}{ll}
\boldsymbol{h}_{11}^{T} & h_{12} \\
H_{21} & \boldsymbol{h}_{22}
\end{array}\right]
$$

with $H_{21}$ an $(n-1) \times(n-1)$ block upper triangular matrix with block partition $\boldsymbol{s}, \boldsymbol{h}_{11}$ and $\boldsymbol{h}_{22}$ vectors of length $n-1$ and $h_{12}$ a scalar.

Definition 2.2 is now extended in an evident manner for matrix pencils. In addition to that, we also introduce the notion of the pole pencil and the pole tuple of a block Hessenberg pencil.

DeFinition 2.3. The $n \times n$ matrix pencil $(A, B)$ is called a block upper Hessenberg pencil with block partition $s=\left(s_{1}, \ldots, s_{m}\right)$ if both $A$ and $B$ are block upper Hessenberg 
matrices with a coinciding block partition,

$$
A=\left[\begin{array}{ll}
\boldsymbol{a}_{11}^{T} & a_{12} \\
A_{21} & \boldsymbol{a}_{22}
\end{array}\right], \quad B=\left[\begin{array}{ll}
\boldsymbol{b}_{11}^{T} & b_{12} \\
B_{21} & \boldsymbol{b}_{22}
\end{array}\right],
$$

$A_{21}, B_{21}(n-1) \times(n-1)$ both block upper triangular matrices having block partition $\boldsymbol{s}=\left(s_{1}, \ldots, s_{m}\right)$. The block upper triangular pencil $\left(A_{21}, B_{21}\right)$ in $(7)$ is called the pole pencil of $(A, B)$. If the pole pencil is regular, the poles $\Xi(A, B)$ are defined as the eigenvalues of the pole pencil, $\Lambda\left(A_{21}, B_{21}\right)$. Since $\left(A_{21}, B_{21}\right)$ admits the partition $\boldsymbol{s}=\left(s_{1}, \ldots, s_{m}\right)$, the pole tuple,

(8) $\Xi(A, B)=\Lambda\left(A_{21}, B_{21}\right)=\left(\Xi^{1}, \ldots, \Xi^{m}\right)=\left(\left\{\xi_{1}^{1}, \ldots, \xi_{s_{1}}^{1}\right\}, \ldots,\left\{\xi_{1}^{m}, \ldots, \xi_{s_{m}}^{m}\right\}\right)$,

admits the same partition. This imposes no specific ordering of the poles within a block but the mutual blocks are ordered.

The previous definitions are illustrated in more detail in the next example.

EXAMPLE 2.4. The $n \times n$ matrices $A, B$ form a block Hessenberg pencil with partition vector $\boldsymbol{s}=\left(s_{1}, \ldots, s_{m}\right)$, if they can be partitioned as:

$$
\left[\begin{array}{ccccc}
\boldsymbol{a}_{1,1}^{T} & \boldsymbol{a}_{1,2}^{T} & \cdots & \boldsymbol{a}_{1, m}^{T} & a_{1, m+1} \\
A_{2,1} & A_{2,2} & \cdots & A_{2, m} & \boldsymbol{a}_{2, m+1} \\
& A_{3,2} & \cdots & A_{3, m} & \boldsymbol{a}_{3, m+1} \\
& & \ddots & \vdots & \vdots \\
& & & A_{m+1, m} & \boldsymbol{a}_{m+1, m+1}
\end{array}\right], \quad\left[\begin{array}{ccccc}
\boldsymbol{b}_{1,1}^{T} & \boldsymbol{b}_{1,2}^{T} & \cdots & \boldsymbol{b}_{1, m}^{T} & b_{1, m+1} \\
B_{2,1} & B_{2,2} & \cdots & B_{2, m} & \boldsymbol{b}_{2, m+1} \\
& B_{3,2} & \cdots & B_{3, m} & \boldsymbol{b}_{3, m+1} \\
& & \ddots & \vdots & \vdots \\
& & & B_{m+1, m} & \boldsymbol{b}_{m+1, m+1}
\end{array}\right],
$$

with all subdiagonal blocks $A_{j+1, j}, B_{j+1, j}$ of size $s_{j} \times s_{j}$ (square) and $s_{1}+\ldots+s_{m}=$ $n-1$. As a specific example, the pencil $(A, B)$ is a $9 \times 9$ block upper Hessenberg pencil with block partition $\boldsymbol{s}=(2,1,3,2)$ if it has the form:
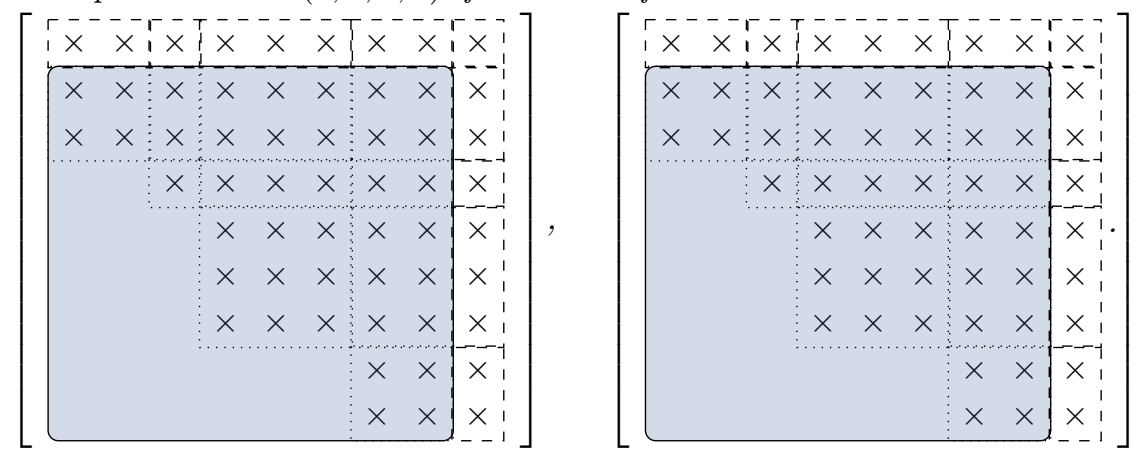

The shaded part of the matrices is the pole pencil which is clearly in block upper triangular form with partition $s=(2,1,3,2)$. The pole tuple is in this case given by,

$$
\Xi(A, B)=\left(\Xi^{1}=\left\{\xi_{1}^{1}, \xi_{2}^{1}\right\}, \Xi^{2}=\left\{\xi_{1}^{2}\right\}, \Xi^{3}=\left\{\xi_{1}^{3}, \xi_{2}^{3}, \xi_{3}^{3}\right\}, \Xi^{4}=\left\{\xi_{1}^{4}, \xi_{2}^{4}\right\}\right) .
$$

Notice that a given block Hessenberg pencil can admit more than one partition. If $(A, B)$ is a block Hessenberg pencil with partition $s=\left(s_{1}, \ldots, s_{k}, s_{k+1}, \ldots, s_{m}\right)$, it also admits the partition $\hat{\boldsymbol{s}}=\left(s_{1}, \ldots, s_{k}+s_{k+1}, \ldots, s_{m}\right)$. Consecutive blocks can be grouped together. Similarly, every $n \times n$ pencil $(A, B)$ can be considered a block Hessenberg pencil with the trivial partition $(n-1)$. We say that $s^{\max }=\left(s_{1}, \ldots, s_{m}\right)$ is the maximal partition of a block Hessenberg pencil if none of its blocks can be split into smaller blocks. For example, a Hessenberg pencil has maximal partition 
$s^{\max }=(1,1, \ldots, 1)$, but admits any other partition. The cumulative partition vector $\boldsymbol{s}^{c}$ of a block Hessenberg pencil with partition $s=\left(s_{1}, \ldots, s_{m}\right)$, is defined as:

$$
\boldsymbol{s}^{c}=\left(s_{1}, s_{1}+s_{2}, \ldots, \sum_{i=1}^{m} s_{i}=n-1\right) .
$$

The last definition we generalize from the Hessenberg pencils of the RQZ method to the block Hessenberg pencils for the multishift, multipole RQZ method is the concept of properness or irreducibility. Properness of the pencil guarantees that there are no obvious options for deflations that split the problem into smaller, independent problems.

Definition 2.5. An $n \times n$ block upper Hessenberg pair $(A, B)$ with partition $s=$ $\left(s_{1}, \ldots, s_{m}\right)$ is said to be proper (or irreducible) if:

I. Its pole pencil is regular;

II. The first block column of $(A, B)$ of size $\left(s_{1}+1\right) \times s_{1}$,

$$
\left[\begin{array}{l}
\boldsymbol{a}_{1,1}^{T} \\
A_{2,1}
\end{array}\right]=\left[\begin{array}{lll}
\boldsymbol{a}_{1} & \ldots & \boldsymbol{a}_{s_{1}}
\end{array}\right], \quad\left[\begin{array}{l}
\boldsymbol{b}_{1,1}^{T} \\
B_{2,1}
\end{array}\right]=\left[\begin{array}{lll}
\boldsymbol{b}_{1} & \ldots & \boldsymbol{b}_{s_{1}}
\end{array}\right], \quad \boldsymbol{a}_{i}, \boldsymbol{b}_{i} \in \mathbb{F}^{s_{1}+1},
$$

satisfies for $i=1, \ldots, s_{1}$,

$$
\mathcal{R}\left(\boldsymbol{a}_{1}, \ldots, \boldsymbol{a}_{i}\right) \neq \mathcal{R}\left(\boldsymbol{b}_{1}, \ldots, \boldsymbol{b}_{i}\right)
$$

III. The last block row of $(A, B)$ of size $s_{m} \times\left(s_{m}+1\right)$,

$$
\left[\begin{array}{ll}
A_{m+1, m} & \boldsymbol{a}_{m+1, m+1}
\end{array}\right]=\left[\begin{array}{c}
\boldsymbol{a}_{s_{m}}^{T} \\
\vdots \\
\boldsymbol{a}_{1}^{T}
\end{array}\right], \quad\left[\begin{array}{ll}
B_{m+1, m} & \boldsymbol{b}_{m+1, m+1}
\end{array}\right]=\left[\begin{array}{c}
\boldsymbol{b}_{s_{m}}^{T} \\
\vdots \\
\boldsymbol{b}_{1}^{T}
\end{array}\right], \quad \boldsymbol{a}_{i}, \boldsymbol{b}_{i} \in \mathbb{F}^{s_{m}+1},
$$

satisfies for $i=1, \ldots, s_{m}$,

$$
\mathcal{R}\left(\boldsymbol{a}_{1}, \ldots, \boldsymbol{a}_{i}\right) \neq \mathcal{R}\left(\boldsymbol{b}_{1}, \ldots, \boldsymbol{b}_{i}\right) .
$$

We remark that condition III is the same as condition II for the pertransposed pencil. This is the pencil obtained after transposition along the anti-diagonal. Furthermore observe that if $(A, B)$ is a Hessenberg pair then the conditions of Definition 2.5 reduce to the same conditions as [6, Definition 2.1]. Conditions II also ensures that property IV of [6, Lemma 2.2] is satisfied within the first block column. We illustrate the notion of (im)properness of a block Hessenberg pencil on a small example to clarify Definition 2.5 .

EXAMPLE 2.6. Consider the $4 \times 4$ real-valued block Hessenberg pencil $(A, B)$ with maximal partition $(2,1)$ given by:

$$
\left[\begin{array}{cccc}
-0.3 & 0.075 & 0.5 & 0.25 \\
0.395 & 0.52 & -0.35 & 2 \\
-0.14 & 0.86 & 1.35 & -0.8 \\
& & 1 & 0.85
\end{array}\right], \quad\left[\begin{array}{cccc}
-0.15 & -0.6 & 0.15 & -1.5 \\
0.16 & 0.94 & -5 & 1.35 \\
-0.12 & -0.08 & -2.4 & -1 \\
& & 0.2 & 1.8
\end{array}\right]
$$

Condition I of Definition 2.5 is satisfied, the pole pencil is regular and the pole tuple of $(A, B)$ is given by:

$$
\Xi=(\{1.5+i \sqrt{15 / 8}, 1.5-i \sqrt{15 / 8}\}, 5) .
$$


The $2 \times 2$ block thus contains a pair of complex conjugate poles. Condition III of Definition 2.5 is also satisfied. For the last block row of $(A, B)$, we clearly have that $\mathcal{R}\left(\left[\begin{array}{ll}1 & 0.85\end{array}\right]\right) \neq \mathcal{R}\left(\left[\begin{array}{ll}0.2 & 1.8\end{array}\right]\right)$. Notice that this implies that we cannot simultaneously create a zero in position $(4,3)$ of both $A$ and $B$ by rotating the last two columns. The block Hessenberg pencil (11) is however improper since Condition II of Definition 2.5 is violated. We have that $\mathcal{R}\left(\boldsymbol{a}_{1}\right) \neq \mathcal{R}\left(\boldsymbol{b}_{1}\right)$, but $\mathcal{R}\left(\boldsymbol{a}_{1}, \boldsymbol{a}_{2}\right)=\mathcal{R}\left(\boldsymbol{b}_{1}, \boldsymbol{b}_{2}\right)$. If we compute an orthonormal basis $Q_{1}$ of $\mathcal{R}\left(\boldsymbol{a}_{1}, \boldsymbol{a}_{2}\right)$ and extend this upto an orthonormal matrix $Q=\left[\begin{array}{ll}Q_{1} & \boldsymbol{q}_{2}\end{array}\right]$, then $(\hat{A}, \hat{B})=Q^{T}(A, B)$ has zero elements in positions $(3,1)$ and $(3,2)$. This deflates the complex conjugate pair of poles in (12) as eigenvalues of the pencil.

The next lemma shows that any proper block Hessenberg pair can be transformed to a proper Hessenberg pair with the same poles.

Lemma 2.7. Given an $n \times n$ proper block Hessenberg pair $(A, B)$ with partition $s=\left(s_{1}, \ldots, s_{m}\right)$ and accordingly partitioned poles $\Xi(A, B)$. Then there exist $n \times n$ unitary block diagonal matrices $Q, Z$,

$$
Q=\operatorname{diag}\left(1, Q_{1}, \ldots, Q_{m}\right) \quad \text { and } \quad Z=\operatorname{diag}\left(Z_{1}, \ldots, Z_{m}, 1\right),
$$

with $Q_{j}, Z_{j}$ unitary matrices of size $s_{j} \times s_{j}$, such that $(\hat{A}, \hat{B})=Q^{*}(A, B) Z$ is a proper Hessenberg pair according to [6, Definition 2.1] with poles $\Xi=\left(\pi_{1}\left(\Xi^{1}\right), \ldots, \pi_{m}\left(\Xi^{m}\right)\right)$. Here, $\pi_{j}\left(\Xi^{j}\right)$ is a permutation of $\xi_{1}^{j}, \ldots, \xi_{s_{j}}^{j}$.

Proof. Since $(A, B)$ is a proper block Hessenberg pencil, the pole pencil is regular and any Schur decomposition of it reduces the block Hessenberg pair to a Hessenberg pair with the same pole tuple as the block Hessenberg pencil. The order of the poles in the Hessenberg pair is, however, dependent on the Schur decomposition.

Moreover, since the pole pencil is a block upper triangular pencil with $m$ blocks, $m$ independent Schur decompositions can be combined as in (13). The pole tuple of the Hessenberg pencil is in this case clearly as described: the poles of the different blocks remain mutually ordered, but within a block any order, or permutation $\pi_{j}$, of the poles is permissible. It remains to verify that conditions II and III of Definition 2.5 are preserved under this transformation. Denote $\hat{Q}=\operatorname{diag}\left(Q_{1}, \ldots, Q_{m}\right)$ and $\hat{Z}=$ $\operatorname{diag}\left(Z_{1}, \ldots, Z_{m}\right)$, with $Q_{j}, Z_{j}$ as in (13). Then,

$$
\begin{aligned}
& \hat{A}=Q^{*} A Z=\operatorname{diag}\left(1, \hat{Q}^{*}\right)\left[\begin{array}{ll}
\boldsymbol{a}_{11}^{T} & a_{12} \\
A_{21} & \boldsymbol{a}_{22}
\end{array}\right] \operatorname{diag}(\hat{Z}, 1)=\left[\begin{array}{cc}
\boldsymbol{a}_{11}^{T} \hat{Z} & a_{12} \\
\hat{Q}^{*} A_{21} \hat{Z} & \hat{Q}^{*} \boldsymbol{a}_{22}
\end{array}\right], \\
& \hat{B}=Q^{*} B Z=\operatorname{diag}\left(1, \hat{Q}^{*}\right)\left[\begin{array}{ll}
\boldsymbol{b}_{11}^{T} & b_{12} \\
B_{21} & \boldsymbol{b}_{22}
\end{array}\right] \operatorname{diag}(\hat{Z}, 1)=\left[\begin{array}{cc}
\boldsymbol{b}_{11}^{T} \hat{Z} & b_{12} \\
\hat{Q}^{*} B_{21} \hat{Z} & \hat{Q}^{*} \boldsymbol{b}_{22}
\end{array}\right] .
\end{aligned}
$$

The first block column of $(\hat{A}, \hat{B})$ is equal to,

$$
\left(\left[\begin{array}{l}
\hat{\boldsymbol{a}}_{1,1}^{T} \\
\hat{A}_{2,1}
\end{array}\right],\left[\begin{array}{l}
\hat{\boldsymbol{b}}_{1,1}^{T} \\
\hat{B}_{2,1}
\end{array}\right]\right)=\left[\begin{array}{ll}
1 & \\
& Q_{1}^{*}
\end{array}\right]\left(\left[\begin{array}{l}
\boldsymbol{a}_{1,1}^{T} \\
A_{2,1}
\end{array}\right],\left[\begin{array}{l}
\boldsymbol{b}_{1,1}^{T} \\
B_{2,1}
\end{array}\right]\right) Z_{1} .
$$

The left and right multiplication of the first block column of $(A, B)$ with unitary matrices clearly preserves condition II of Definition 2.5. Also condition III is preserved under the equivalence transformation of (13). This directly implies that the resulting Hessenberg pair is also proper according to [6, Definition 2.1].

We remark that since a real-valued block Hessenberg pencils can have complex conjugate pairs of poles, its proper Hessenberg form of Lemma 2.7 will be complexvalued. 
2.2. Rational Krylov and block Hessenberg pencils. In this section we study the structure of rational Krylov subspaces generated by proper block Hessenberg matrices. These results are useful for the analysis of the pole introduction operation introduced in Section 2.3 and to study uniqueness of a multishift, multipole RQZ step in Section 3. We give a brief introduction to rational Krylov matrices and subspaces for the sake of completeness. For a more detailed overview of this subject matter, we refer the interested reader to [6] and the references therein.

We use the same notational conventions as in [6]. Given a matrix pair $(A, B) \in$ $\mathbb{F}^{n \times n}$, shift $\varrho=\mu / \nu \in \overline{\mathbb{C}}$ and pole $\xi=\alpha / \beta \in \overline{\mathbb{C}} \backslash \Lambda$, we define the following elementary rational matrices,

$$
\begin{aligned}
M(\varrho, \xi) & =(\nu A-\mu B)(\beta A-\alpha B)^{-1}, \\
N(\varrho, \xi) & =(\beta A-\alpha B)^{-1}(\nu A-\mu B) .
\end{aligned}
$$

Notice that the matrices $M(\varrho, \xi)$ and $N(\varrho, \xi)$ represent an entire class of matrices that are all nonzero scalar multiple of each other. Every representative is fine as the results we present are scale invariant. The elementary rational matrices satisfy a few basic properties. The inverse $M(\varrho, \xi)^{-1}$ is defined if $\varrho \notin \Lambda$ and is equal to $M(\xi, \varrho)$. They are commutative, $M\left(\varrho_{1}, \xi_{1}\right) M\left(\varrho_{2}, \xi_{2}\right)=M\left(\varrho_{2}, \xi_{2}\right) M\left(\varrho_{1}, \xi_{1}\right)$, and they can be merged together, $M\left(\varrho, \xi_{1}\right) M\left(\xi_{1}, \xi_{2}\right)=M\left(\varrho, \xi_{2}\right)$, if a pole and shift are equal. Analogous results hold for $N(\varrho, \xi)$.

These elementary rational matrices are used to construct rational Krylov matrices generated by a regular matrix pair, a starting vector, and a tuple of poles.

Definition 2.8. Let $A, B \in \mathbb{F}^{n \times n}$ form a regular matrix pair, $\boldsymbol{v} \in \mathbb{F}^{n}$ a nonzero vector, $k \leq n, \Xi=\left(\xi_{1}, \ldots, \xi_{k-1}\right)$ a tuple of poles that are distinct from the eigenvalues, and $P=\left(\varrho_{1}, \ldots, \varrho_{k-1}\right) \subset \overline{\mathbb{C}}$ a tuple of shifts distinct from the poles. The corresponding rational Krylov matrices are defined as:

$$
\begin{aligned}
K_{k}^{r a t}(A, B, \boldsymbol{v}, \Xi, P) & =\left[\boldsymbol{v}, M\left(\varrho_{1}, \xi_{1}\right) \boldsymbol{v}, M\left(\varrho_{2}, \xi_{2}\right) M\left(\varrho_{1}, \xi_{1}\right) \boldsymbol{v}, \ldots,\left(\prod_{i=1}^{k-1} M\left(\varrho_{i}, \xi_{1}\right)\right) \boldsymbol{v}\right], \\
L_{k}^{r a t}(A, B, \boldsymbol{v}, \Xi, P) & =\left[\boldsymbol{v}, N\left(\varrho_{1}, \xi_{1}\right) \boldsymbol{v}, N\left(\varrho_{2}, \xi_{2}\right) N\left(\varrho_{1}, \xi_{1}\right) \boldsymbol{v}, \ldots,\left(\prod_{i=1}^{k-1} N\left(\varrho_{i}, \xi_{i}\right)\right) \boldsymbol{v}\right] .
\end{aligned}
$$

The column spaces of these matrices span the rational Krylov subspaces.

Definition 2.9. The rational Krylov subspaces $\mathcal{K}_{k}^{\text {rat }}$ and $\mathcal{L}_{k}^{\text {rat }}, k \leq n$, associated with the $n \times n$ regular pair $(A, B)$, a nonzero vector $\boldsymbol{v} \in \mathbb{F}^{n}$, and pole tuple $\Xi=$ $\left(\xi_{1}, \ldots, \xi_{k-1}\right)$ distinct from the eigenvalues, are defined as,

$$
\begin{aligned}
& \mathcal{K}_{k}^{r a t}(A, B, \boldsymbol{v}, \Xi) \equiv \mathcal{R}\left(K_{k}^{r a t}(A, B, \boldsymbol{v}, \Xi, \mathrm{P})\right)=\prod_{i=1}^{k-1} M\left(\hat{\varrho}, \xi_{i}\right) \cdot \mathcal{K}_{k}(M(\check{\varrho}, \hat{\varrho}), \boldsymbol{v}), \\
& \mathcal{L}_{k}^{r a t}(A, B, \boldsymbol{v}, \Xi) \equiv \mathcal{R}\left(L_{k}^{r a t}(A, B, \boldsymbol{v}, \Xi, \mathrm{P})\right)=\prod_{i=1}^{k-1} N\left(\hat{\varrho}, \xi_{i}\right) \cdot \mathcal{K}_{k}(N(\check{\varrho}, \hat{\varrho}), \boldsymbol{v}),
\end{aligned}
$$

where the shift tuple $\mathrm{P}$ is freely chosen in agreement with Definition 2.8, $\hat{\varrho}$ is a shift different from the eigenvalues and poles, and $\varrho$ is an alternative shift different from $\hat{\varrho}$. 
The first equality in (16) defines the rational Krylov subspaces, the second equality repeats [6, Lemma 5.6.II]. This result shows that rational Krylov subspaces are shift invariant as they are independent of the choice shifts $\mathrm{P}$.

The following theorem is a block generalization of [6, Theorem 5.6] and shows that the rational Krylov subspaces $\mathcal{K}^{\text {rat }}$ and $\mathcal{L}^{\text {rat }}$ have a specific structure if they are generated from a proper block Hessenberg pair.

TheOREM 2.10. Given an $n \times n$ proper block Hessenberg pair $(A, B)$ with partition $s=\left(s_{1}, \ldots, s_{m}\right)$, cumulative partition $s^{c}$, poles $\Xi=\left(\Xi^{1}, \ldots, \Xi^{m}\right)$ with $\Xi^{i}=\left\{\xi_{1}^{i}, \ldots \xi_{s_{i}}^{i}\right\}$ that are all different from the eigenvalues. Then for $j=0,1, \ldots, m$,

$$
\mathcal{K}_{s_{j}^{c}+1}^{r a t}\left(A, B, \boldsymbol{e}_{1},\left(\Xi^{1}, \ldots, \Xi^{j}\right)\right)=\mathcal{E}_{s_{j}^{c}+1},
$$

with $s_{0}^{c} \equiv 0$. While for $j=1, \ldots, m$,

$$
\mathcal{L}_{s_{j}^{c}}^{r a t}\left(A, B, \boldsymbol{z}_{1},\left(\breve{\Xi}^{1}, \Xi^{2}, \ldots, \Xi^{j}\right)\right)=\mathcal{E}_{s_{j}^{c}},
$$

with $\breve{\Xi}^{1}=\left\{\xi_{1}^{1}, \ldots, \xi_{s_{1}-1}^{1}\right\}$, and $\boldsymbol{z}_{1}$ the right eigenvector of the pole pencil corresponding with pole $\xi_{s_{1}}^{1}$. Here $\xi_{s_{1}}^{1}$ can be any of the poles in $\Xi^{1}$.

Proof. We rely on the transformation $(\hat{A}, \hat{B})=Q^{*}(A, B) Z$ from proper block Hessenberg pencil $(A, B)$ to proper Hessenberg pencil $(\hat{A}, \hat{B})$ as defined in Lemma 2.7. Denote with $\hat{\Xi}=\left(\xi_{1}, \ldots, \xi_{n-1}\right)$ the pole tuple of the proper Hessenberg pair $(\hat{A}, \hat{B})$ after renumbering. Note that, by construction, in (13), $\boldsymbol{q}_{1}=\boldsymbol{e}_{1}$ and denote $\hat{M}(\varrho, \xi)=$ $Q^{*} M(\varrho, \xi) Q$ as the elementary rational matrix of $(14)$ in terms of $(\hat{A}, \hat{B})$. Further we apply [6, Theorem 5.6] to $(\hat{A}, \hat{B})$ such that for $k$ from 1 to $n$,

$$
\begin{aligned}
\mathcal{E}_{k} & =\mathcal{K}_{k}^{\mathrm{rat}}\left(\hat{A}, \hat{B}, \boldsymbol{e}_{1},\left(\xi_{1}, \ldots, \xi_{k-1}\right)\right)=\prod_{i=1}^{k-1} \hat{M}\left(\hat{\varrho}, \xi_{i}\right) \cdot \mathcal{K}_{k}\left(\hat{M}(\check{\varrho}, \hat{\varrho}), \boldsymbol{e}_{1}\right) \\
& =Q^{*} \prod_{i=1}^{k-1} M\left(\hat{\varrho}, \xi_{i}\right) \cdot \mathcal{K}_{k}\left(M(\check{\varrho}, \hat{\varrho}), \boldsymbol{q}_{1}\right)=Q^{*} \mathcal{K}_{k}^{\mathrm{rat}}\left(A, B, \boldsymbol{e}_{1},\left(\xi_{1}, \ldots, \xi_{k-1}\right)\right) .
\end{aligned}
$$

Multiplying both sides of this equation with $Q$ and using that (13) implies that $Q \mathcal{E}_{k}=$ $\mathcal{E}_{k}$ for $k \in\left\{1, s_{1}^{c}+1, \ldots, s_{m}^{c}+1=n\right\}$, proving thereby the first part of the theorem. The second part of the theorem can be proven in an analogous manner. Denote $\hat{N}(\varrho, \xi)=Z^{*} N(\varrho, \xi) Z$ as the second elementary rational matrix of (14) in terms of $(\hat{A}, \hat{B})$ and apply again $[6$, Theorem 5.6$]$ to $(\hat{A}, \hat{B})$ such that for $k$ from 1 to $n-1$,

$$
\begin{aligned}
\mathcal{E}_{k} & =\mathcal{L}_{k}^{\mathrm{rat}}\left(\hat{A}, \hat{B}, \boldsymbol{e}_{1},\left(\xi_{2}, \ldots, \xi_{k}\right)\right)=\prod_{i=2}^{k} \hat{N}\left(\hat{\varrho}, \xi_{i}\right) \cdot \mathcal{K}_{k}\left(\hat{N}(\varrho \varrho \varrho \varrho), \boldsymbol{e}_{1}\right) \\
& =Z^{*} \prod_{i=2}^{k} N\left(\hat{\varrho}, \xi_{i}\right) \cdot \mathcal{K}_{k}\left(N(\varrho \varrho \varrho \varrho), \boldsymbol{z}_{1}\right)=Z^{*} \mathcal{L}_{k}^{\mathrm{rat}}\left(A, B, \boldsymbol{z}_{1},\left(\xi_{2}, \ldots, \xi_{k}\right)\right) .
\end{aligned}
$$

Now multiply both sides with $Z$ and again use (13) to show that $Z \mathcal{E}_{k}=\mathcal{E}_{k}$ for $k \in$ $\left\{s_{1}^{c}, \ldots, s_{m}^{c}, n\right\}$ and the second part of the theorem is proven. Recall from Lemma 2.7 that $\boldsymbol{z}_{1}$ is the right eigenvector of the pole pencil related to $\xi_{1}$ and that $\xi_{1}$ can be any of the poles of $\Xi^{1}$ since for any $\xi_{j}^{1}$ there exists a block Schur decomposition (13) that places $\xi_{j}^{1}$ as the first pole in the Hessenberg pencil $(\hat{A}, \hat{B})$. 
2.3. Manipulating poles of block Hessenberg pencils. Throughout this section, the pencil $(A, B)$ is assumed to be an $n \times n$ proper block Hessenberg pencil with maximal partition $\boldsymbol{s}=\left(s_{1}, \ldots, s_{m}\right)$ and pole tuple $\Xi=\left(\Xi^{1}, \ldots, \Xi^{m}\right)$, where $\Xi^{j}=\left\{\xi_{1}^{j}, \ldots, \xi_{s_{j}}^{j}\right\}$. All poles are assumed different from the eigenvalues.

We review two different operations to change the pole tuple $\Xi$. The first operation changes the first or last $\ell$ poles of the pencil, the second operation swaps two adjacent pole blocks $\Xi^{i}$ and $\Xi^{i+1}$.

Changing poles at the boundary. The first $\ell=s_{1}+\ldots+s_{i}=s_{i}^{c}$ poles in the first $i$ pole blocks $\Xi^{1}, \ldots, \Xi^{i}$ can be changed to $\ell$ new poles $\mathrm{P}=\left\{\varrho_{1}, \ldots, \varrho_{\ell}\right\}$. We assume $\mathrm{P}$ distinct from the original poles. For this purpose consider the vector,

$$
\boldsymbol{x}=\gamma \prod_{j=1}^{\ell} M\left(\varrho_{j}, \xi_{j}\right) \boldsymbol{e}_{1},
$$

with $\xi_{1}, \ldots, \xi_{\ell}$ the poles of $\Xi^{1}, \ldots, \Xi^{i}$. The following procedure can be used to compute $\boldsymbol{x}$,

$$
\begin{aligned}
& \boldsymbol{x} \leftarrow \boldsymbol{e}_{1} \\
& \text { for } j=1, \ldots, \ell \\
& {\left[\boldsymbol{x} \leftarrow \gamma_{j} M\left(\varrho_{j}, \xi_{j}\right) \boldsymbol{x}\right.}
\end{aligned}
$$

The scalars $\gamma_{j}$ can be chosen as some suitable scaling factors. Now compute a unitary matrix $Q$ such that,

$$
Q^{*} \boldsymbol{x}=\alpha \boldsymbol{e}_{1} .
$$

We claim that the new poles $P$ are introduced in the block Hessenberg pair by updating $(\hat{A}, \hat{B})=Q^{*}(A, B)$. Specifically, $(\hat{A}, \hat{B})$ is a block Hessenberg pair with maximal partition $\hat{\boldsymbol{s}}=\left(\ell, s_{i+1}, \ldots, s_{m}\right)$ and poles $\hat{X} i=\left(\mathrm{P}, \Xi^{i+1}, \ldots, \Xi^{m}\right)$.

From (16) and Theorem 2.10 we have that,

$$
\boldsymbol{x} \in \mathcal{K}_{\ell+1}^{\mathrm{rat}}\left(A, B, \boldsymbol{e}_{1}, \Xi\right)=\mathcal{E}_{\ell+1} .
$$

This implies that $Q$ in (21) is of the form $\operatorname{diag}\left(Q_{\ell+1}, I\right)$, with $Q_{\ell+1}$ an $(\ell+1) \times(\ell+1)$ unitary matrix. It follows that the first block $\hat{\Xi}^{1}$ in $(\hat{A}, \hat{B})$ is indeed of size $\ell$. Furthermore, for $j=0,1, \ldots, m-i+1$,

$$
\begin{aligned}
& \mathcal{K}_{\hat{s}_{j}^{c}+1}^{\mathrm{rat}}\left(\hat{A}, \hat{B}, \boldsymbol{e}_{1},\left(\mathrm{P}, \Xi^{i+1}, \ldots, \Xi^{m}\right)\right)=\prod_{k=1}^{\hat{s}_{j}^{c}} \hat{M}\left(\hat{\varrho}, \hat{\xi}_{k}\right) \cdot \mathcal{K}_{\hat{s}_{j}^{c}+1}\left(\hat{M}(\check{\varrho}, \hat{\varrho}), \boldsymbol{e}_{1}\right)
\end{aligned}
$$

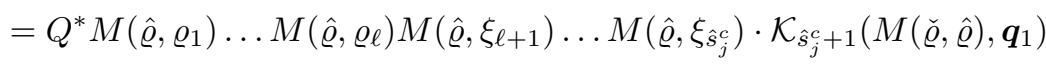

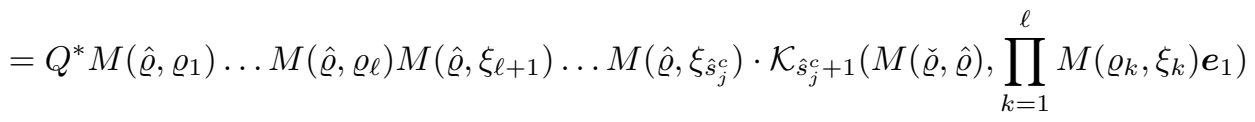

$$
\begin{aligned}
& =Q^{*} M\left(\varrho \hat{\varrho}, \xi_{1}\right) \ldots M\left(\hat{\varrho}, \xi_{\ell}\right) M\left(\hat{\varrho}, \xi_{\ell+1}\right) \ldots M\left(\hat{\varrho}, \xi_{\hat{s}_{j}^{c}}\right) \cdot \mathcal{K}_{\hat{s}_{j}^{c}+1}\left(M(\check{\varrho}, \hat{\varrho}), \boldsymbol{e}_{1}\right) \\
& =Q^{*} \mathcal{K}_{\hat{s}_{j}^{c}+1}^{\mathrm{rat}}\left(A, B, \boldsymbol{e}_{1},\left(\Xi^{1}, \ldots, \Xi^{i}, \Xi^{i+1}, \ldots, \Xi^{m}\right)\right) \\
& =Q^{*} \mathcal{E}_{\hat{s}_{j}^{c}+1}=\mathcal{E}_{\hat{s}_{j}^{c}+1} \text {. }
\end{aligned}
$$

In the first equality we used (16), we applied $\hat{M}=Q^{*} M Q$ in the second equality, and combined (19) and (21) to get $\boldsymbol{q}_{1}=\prod_{k=1}^{\ell} M\left(\varrho_{k}, \xi_{k}\right) \boldsymbol{e}_{1}$ in the third equality. 
The fourth equality uses the commutativity of the $M$ matrices and the property that $M\left(\hat{\varrho}, \varrho_{k}\right) M\left(\varrho_{k}, \xi_{k}\right)$ can be merged to $M\left(\varrho \hat{\varrho}, \xi_{k}\right)$. This results in the rational Krylov subspace of the original pencil with the original poles in the fifth equality and by Theorem 2.10 we know that this is equal to $\mathcal{E}_{\hat{s}_{j}^{c}+1}$. Finally, since $Q$ has a block diagonal structure, it does not affect the $\mathcal{E}_{\hat{s}_{j}^{c}+1}$ for the given sizes. It is clear that $(\hat{A}, \hat{B})$ is a proper block Hessenberg pencil with partition $\hat{s}=\left(\ell, s_{i+1}, \ldots, s_{m}\right)$ by construction. The last poles are unchanged by the block diagonal structure of $Q$ and the first $\ell$ poles are changed to $\mathrm{P}$ which follows from the uniqueness of block Hessenberg pencils, see Theorem 3.3.

We remark that in order to compute the vector $\boldsymbol{x}$ in (20), $\ell$ shifted linear systems need to be solved as $M\left(\varrho_{i}, \xi_{i}\right)=\left(\nu_{i} A-\mu_{i} B\right)\left(\beta_{i} A-\alpha_{i} B\right)^{-1}$. These linear systems are essentially of size $\ell$ because $\left(\beta_{\ell} A-\alpha_{\ell} B\right)^{-1}$ is a block upper triangular matrix with a leading block of size $\ell \times \ell$. This limits the computational cost of computing $\boldsymbol{x}$ to $O\left(\ell^{4}\right)$, which is small as long as $\ell \ll n$. It also follows that the vector $\boldsymbol{x}$ can be computed even when poles in $\Xi^{1}, \ldots, \Xi^{i}$ are equal to eigenvalues of the pencil. Properness ensures that the leading $\ell \times \ell$ block is nonsingular.

The last $\ell$ poles in the last $i$ blocks $\Xi^{m-i+1}, \ldots, \Xi^{m}$ of $(A, B)$ can be changed to $\mathrm{P}=\left\{\varrho_{1}, \ldots, \varrho_{\ell}\right\}$ in a similar fashion. We compute first the row vector,

$$
\boldsymbol{x}^{T}=\gamma \boldsymbol{e}_{n}^{T} \prod_{j=m-\ell+1}^{m} N\left(\varrho_{j}, \xi_{j}\right),
$$

and then a unitary matrix $Z=\operatorname{diag}\left(I, Z_{\ell+1}\right)$ such that $\boldsymbol{x}^{T} Z=\alpha \boldsymbol{e}_{n}^{T}$. The pencil $(\hat{A}, \hat{B})=(A, B) Z$ then becomes a block Hessenberg pencil with pole tuple $\left(\Xi^{1}, \ldots\right.$, $\left.\Xi^{m-i}, \mathrm{P}\right)$.

We remark that if $(A, B)$ is a real-valued pencil and the poles and shifts considered in (19) and (23) are both closed under complex conjugation, then the vectors $\boldsymbol{x}$ and $\boldsymbol{x}^{T}$ and consequently the matrices $Q$ and $Z$ are also real-valued. This follows from the commutativity property in combination with the property that $M(\bar{\varrho}, \bar{\xi})=\overline{M(\varrho, \xi)}$ for real-valued pencils. We have,

$$
\overline{M(\varrho, \xi) M(\bar{\varrho}, \bar{\xi})}=\overline{M(\bar{\varrho}, \bar{\xi}) M(\varrho, \xi)}=M(\varrho, \xi) M(\bar{\varrho}, \bar{\xi})
$$

so $M(\varrho, \xi) M(\bar{\varrho}, \bar{\xi})$ is a real-valued matrix if $A$ and $B$ are real-valued.

Swapping adjacent pole blocks. A second operation to change the pole tuple of a block Hessenberg pencil is swapping two consecutive blocks in the pole pencil. Swapping block $i$ with block $i+1$ requires the computation of a unitary equivalence essentially of size $\left(s_{i}+s_{i+1}\right) \times\left(s_{i}+s_{i+1}\right)$ that updates the pencil $(\hat{A}, \hat{B})=Q^{*}(A, B) Z$ in such a way that the new pole tuple and partition vector are given by,

$$
\begin{aligned}
\hat{\Xi} & =\left(\Xi^{1}, \ldots, \Xi^{i-1}, \Xi^{i+1}, \Xi^{i}, \Xi^{i+2}, \ldots, \Xi^{m}\right), \\
\hat{\boldsymbol{s}} & =\left(s_{1}, \ldots, s_{i-1}, s_{i+1}, s_{i}, s_{i+2}, \ldots, s_{m}\right) .
\end{aligned}
$$

This problem is equivalent to reordering eigenvalues in the generalized Schur form. Two different approaches to solve this problem have been proposed in the literature. The first approach, studied by Kågström [11, 13], requires the solution of a coupled Sylvester equation. This method is applicable for general blocksizes $s_{i}, s_{i+1}$. The second approach, studied by Van Dooren [17], is a direct method that relies on the computation of a right eigenvector of a pole in block $i+1$. This method has been studied for swapping a block of dimension $1 \times 1$ or $2 \times 2$ with a block of dimension $1 \times 1$, or vice versa. 
2.4. Multishift, multipole RQZ step. Combining the operations from the previous subsection, we propose following three step procedure as the generic multishift, multipole RQZ step.

I. Starting from a proper block Hessenberg pencil with pole tuple $\Xi=\left(\Xi^{1}, \ldots, \Xi^{m}\right)$ and partition $s=\left(s_{1}, \ldots, s_{m}\right)$, select or compute $\ell=s_{1}+\ldots+s_{i}=s_{i}^{c}$ shifts P. Introduce the shifts in the block Hessenberg pencil by computing the vector $\boldsymbol{x}$ via (20) and the orthonormal matrix $Q$ via (21) and updating the pencil accordingly. The pencil now has pole tuple $\Xi=\left(\mathrm{P}, \Xi^{i+1}, \ldots, \Xi^{m}\right)$ and partition vector $s=\left(\ell, s_{i+1}, \ldots, s_{m}\right)$.

II. Repeatedly use the swapping procedure to construct a unitary equivalence that moves the shifts $\mathrm{P}$ to the last pole block. This changes the pole tuple to $\Xi=$ $\left(\Xi^{i+1}, \ldots, \Xi^{m}, \mathrm{P}\right)$ and the partition vector to $s=\left(s_{i+1}, \ldots, s_{m}, \ell\right)$.

III. Compute or select $\ell$ new poles $\Xi^{m+1}$ and introduce them at the end of the pencil to change the pole tuple to $\Xi=\left(\Xi^{i+1}, \ldots, \Xi^{m}, \Xi^{m+1}\right)$.

These three steps constitute a single multishift, multipole RQZ sweep. After every sweep, the properness of the pencil is checked and the problem is split into independent subproblems wherever possible.

The multishift QZ method is a special case of this algorithm where the pencil initially has pole tuple $(\infty, \ldots, \infty)$ and partition $(1, \ldots, 1)$ and where this form is always restored in step III of the algorithm. The single shift RQZ method is also a special case of this algorithm.

In Sections 4 and 5 we address a couple of numerical challenges that make the multishift, multipole RQZ step stable and efficient in finite precision arithmetic. First, Section 3 provides further theoretical foundation for the implicit approach.

3. Uniqueness and convergence. In this section we motivate the implicit approach used in the multishift, multipole RQZ step in the form of an implicit $Q$ theorem for block Hessenberg pencils. We also discuss the subspace iteration that is implicitly performed during the multishift, multipole RQZ step.

The following lemma first extends the essential uniqueness of the $\mathrm{QR}$ factorization to a form of essential uniqueness in the factorization of a matrix as a product of a unitary matrix and a block upper triangular matrix.

Lemma 3.1. Given a nonsingular $n \times n$ matrix $A$ and consider $A=\hat{Q} \hat{R}_{s}, A=$ $\check{Q}_{L_{s}}$ two block $Q R$ factorizations where $\hat{Q}, \check{Q}$ are unitary matrices and $\hat{R}_{s}, \check{R}_{s}$ are block upper triangular matrices with the same partition $s=\left(s_{1}, \ldots, s_{m}\right)$. Then $\hat{Q}=\check{Q} D_{s}$ with $D_{s}$ a unitary block diagonal matrix with an identical partition $s$ as $\hat{R}_{\boldsymbol{s}}$ and $\check{R}_{\boldsymbol{s}}$.

Proof. From $\hat{Q} \hat{R}_{s}=\check{Q}_{\mathrm{R}_{s}}$ it follows that, $\check{Q}^{*} \hat{Q}=\check{R}_{s} \hat{R}_{s}^{-1}=\tilde{R}_{s}=D_{s}$, with $\tilde{R}_{s}$ a unitary block upper triangular matrix with partition $s$. The only unitary block upper triangular matrices are block diagonal matrices $D_{\boldsymbol{s}}$.

Before presenting the implicit $\mathrm{Q}$ theorem, we first give this direct corollary of Theorem 2.10 that considers the structure of rational Krylov matrices instead of the subspaces. This is the block generalization of [6, Corollary 5.7].

Corollary 3.2. Given an $n \times n$ proper block Hessenberg pair $(A, B)$ with partition $\boldsymbol{s}=\left(s_{1}, \ldots, s_{m}\right)$ and poles $\Xi=\left(\Xi^{1}, \ldots, \Xi^{m}\right)$ that are different from the eigenvalues. Then for a tuple of shifts $\mathrm{P}$ different from the poles, $K_{n}^{\text {rat }}\left(A, B, \boldsymbol{e}_{1}, \Xi, \mathrm{P}\right)$ is a full rank $n \times n$ block upper triangular matrix with partition $\left(1, s_{1}, s_{2}, \ldots, s_{m}\right)$. While, $L_{n-1}^{\text {rat }}\left(A, B, \boldsymbol{z}_{1},\left(\breve{\Xi}^{1}, \Xi^{2}, \ldots, \Xi^{m}\right), \mathrm{P}\right)$ is a full rank $n \times n-1$ block upper triangular matrix with partition $\left(s_{1}, s_{2}, \ldots, s_{m}\right)$. Here $\boldsymbol{z}_{1}$ and $\breve{\Xi}^{1}$ are chosen as described in Theorem 2.10 . 
We are now ready to state the block implicit Q theorem.

THeOREM 3.3. Let $(A, B)$ be a regular matrix pair and let $\hat{Q}, \check{Q}, \hat{Z}, \check{Z}$ be unitary matrices with $\hat{Q} \boldsymbol{e}_{1}=\sigma \check{Q} \boldsymbol{e}_{1},|\sigma|=1$, such that,

$$
(\hat{A}, \hat{B})=\hat{Q}^{*}(A, B) \hat{Z} \quad \text { and } \quad(\check{A}, \check{B})=\check{Q}^{*}(A, B) \check{Z},
$$

are both proper block Hessenberg pairs with the same partition $\left(s_{1}, \ldots, s_{m}\right)$ and poles $\Xi=\left(\Xi^{1}, \ldots, \Xi^{m}\right)$ different from the eigenvalues. Then the pairs $(\hat{A}, \hat{B})$ and $(\check{A}, \check{B})$ are identical up to multiplication with two unitary block diagonal matrices,

$$
\hat{A}=D_{1}^{*} \check{A} D_{2} \quad \text { and } \quad \hat{B}=D_{1}^{*} \check{B} D_{2},
$$

with $D_{1}$ having partition $\left(1, s_{1}, \ldots, s_{m}\right)$ and $D_{2}$ having partition $\left(s_{1}, \ldots, s_{m}, 1\right)$.

Proof. Corollary 3.2 states that $K_{n}^{\mathrm{rat}}\left(\hat{A}, \hat{B}, \boldsymbol{e}_{1}, \Xi, \mathrm{P}\right)$ and $K_{n}^{\mathrm{rat}}\left(\check{A}, \check{B}, \boldsymbol{e}_{1}, \Xi, \mathrm{P}\right)$ are both block upper triangular matrices of full rank with block partition $\left(1, s_{1}, \ldots, s_{m}\right)$. We thus have,

$$
\begin{aligned}
& \hat{Q} K_{n}^{\mathrm{rat}}\left(\hat{A}, \hat{B}, \boldsymbol{e}_{1}, \Xi, \mathrm{P}\right) \\
= & \hat{Q}\left[\boldsymbol{e}_{1}, \hat{M}\left(\varrho_{1}, \xi_{1}\right) \boldsymbol{e}_{1}, \ldots,\left(\prod_{i=1}^{n-1} \hat{M}\left(\varrho_{i}, \xi_{i}\right)\right) \boldsymbol{e}_{1}\right] \\
= & \hat{Q}\left[\boldsymbol{e}_{1}, \hat{Q}^{*} M\left(\varrho_{1}, \xi_{1}\right) \hat{Q} \boldsymbol{e}_{1}, \ldots, \hat{Q}^{*}\left(\prod_{i=1}^{n-1} M\left(\varrho_{i}, \xi_{i}\right)\right) \hat{Q} \boldsymbol{e}_{1}\right] \\
= & {\left[\hat{\boldsymbol{q}}_{1}, M\left(\varrho_{1}, \xi_{1}\right) \hat{\boldsymbol{q}}_{1}, \ldots,\left(\prod_{i=1}^{n-1} M\left(\varrho_{i}, \xi_{i}\right)\right) \hat{\boldsymbol{q}}_{1}\right] } \\
= & \sigma\left[\check{\boldsymbol{q}}_{1}, M\left(\varrho_{1}, \xi_{1}\right) \check{\boldsymbol{q}}_{1}, \ldots,\left(\prod_{i=1}^{n-1} M\left(\varrho_{i}, \xi_{i}\right)\right) \check{\boldsymbol{q}}_{1}\right] \\
= & \sigma \check{Q} K_{n}^{\mathrm{rat}}\left(\check{A}, \check{B}, \boldsymbol{e}_{1}, \Xi, \mathrm{P}\right) .
\end{aligned}
$$

From Lemma 3.1 we have that this equality between two block QR factorizations implies that $\hat{Q}=\check{Q} D_{\left(1, s_{1}, \ldots, s_{m}\right)}$. For the relation between $\hat{Z}$ and $\check{Z}$, consider,

$$
(\hat{\hat{A}}, \hat{B})=\hat{Q}^{*}(\hat{A}, \hat{B}) \hat{Z}, \quad \text { and }, \quad(\check{A}, \check{\check{B}})=\check{Q}^{*}(\check{A}, \check{B}) \check{Z}
$$

both reductions of the block Hessenberg pencils to a proper Hessenberg pencil as defined in Lemma 2.7 and assume, without loss of generality, that $\xi_{s_{1}}^{1}$ is the first pole in both $(\hat{\hat{A}}, \hat{\hat{B}})$ and $(\check{A}, \check{B})$. Thus $\hat{\boldsymbol{z}}_{1}$ is the right eigenvector of the pole pencil of $(\hat{\hat{A}}, \hat{\hat{B}})$ associated with the eigenvalue $\xi_{s_{1}}^{1}$ and the same holds for $\check{\check{z}}_{1}$ and $(\check{A}, \check{B})$. This implies,

$$
\hat{\hat{Q}}^{*}\left(\hat{A}-\xi_{s_{1}}^{1} \hat{B}\right) \hat{\hat{z}}_{1}=\hat{\gamma} \boldsymbol{e}_{1}, \quad \text { and, } \quad \check{Q}^{*}\left(\check{A}-\xi_{s_{1}}^{1} \check{B}\right) \check{z}_{1}=\check{\gamma} \boldsymbol{e}_{1},
$$

by the proper Hessenberg structure of $(\hat{\hat{A}}, \hat{\hat{B}})$ and $(\check{\mathscr{A}}, \check{\check{B}})$. Since by $(13), \hat{\hat{Q}} \boldsymbol{e}_{1}=\check{Q} \boldsymbol{e}_{1}=$ $\boldsymbol{e}_{1}$, we also have,

$$
\left(\hat{A}-\xi_{s_{1}}^{1} \hat{B}\right) \hat{\boldsymbol{z}}_{1}=\hat{\gamma} \boldsymbol{e}_{1}, \quad \text { and }, \quad\left(\check{A}-\xi_{s_{1}}^{1} \check{B}\right) \check{\check{z}}_{1}=\check{\gamma} \boldsymbol{e}_{1},
$$

Thus,

$$
\hat{Q}^{*}\left(A-\xi_{s_{1}}^{1} B\right) \hat{Z} \hat{\boldsymbol{z}}_{1}=\hat{\gamma} \boldsymbol{e}_{1}, \quad \text { and }, \quad \check{Q}^{*}\left(A-\xi_{s_{1}}^{1} B\right) \check{Z} \check{\boldsymbol{z}}_{1}=\check{\gamma} \boldsymbol{e}_{1} .
$$


Using, $\hat{Q}=\check{Q} D_{\left(1, s_{1}, \ldots, s_{m}\right)}, D_{\left(1, s_{1}, \ldots, s_{m}\right)} \boldsymbol{e}_{1}=\sigma \boldsymbol{e}_{1}$, we get that,

$$
\begin{aligned}
& \hat{Z} \hat{\boldsymbol{z}}_{1}=\hat{\gamma}\left(A-\xi_{s_{1}}^{1} B\right)^{-1} \check{Q} D_{\left(1, s_{1}, \ldots, s_{m}\right)} \boldsymbol{e}_{1}=\sigma \hat{\gamma}\left(A-\xi_{s_{1}}^{1} B\right)^{-1} \check{Q} \boldsymbol{e}_{1} \\
& \check{Z} \check{\check{z}}_{1}=\check{\gamma}\left(A-\xi_{s_{1}}^{1} B\right)^{-1} \check{Q} \boldsymbol{e}_{1},
\end{aligned}
$$

from which we conclude that $\hat{Z} \hat{\boldsymbol{z}}_{1}=\tilde{\sigma} \check{Z} \check{\boldsymbol{z}}_{1}$ for some $\tilde{\sigma}$ with $|\tilde{\sigma}|=1$. Now use this result in combination with Corollary 3.2 ,

$$
\begin{aligned}
& \hat{Z} L_{n-1}^{\mathrm{rat}}\left(\hat{A}, \hat{B}, \hat{\boldsymbol{z}}_{1}, \Xi, \mathrm{P}\right) \\
= & \hat{Z}\left[\hat{\boldsymbol{z}}_{1}, \hat{N}\left(\varrho_{1}, \xi_{1}\right) \hat{\boldsymbol{z}}_{1}, \ldots,\left(\prod_{i=2}^{n-1} \hat{N}\left(\varrho_{i}, \xi_{i}\right)\right) \hat{\boldsymbol{z}}_{1}\right] \\
= & {\left[\hat{Z} \hat{\boldsymbol{z}}_{1}, N\left(\varrho_{1}, \xi_{1}\right) \hat{Z} \hat{\boldsymbol{z}}_{1}, \ldots,\left(\prod_{i=2}^{n-1} N\left(\varrho_{i}, \xi_{i}\right)\right) \hat{Z} \hat{\boldsymbol{z}}_{1}\right] } \\
= & \tilde{\sigma}\left[\check{Z} \check{\check{z}}_{1}, N\left(\varrho_{1}, \xi_{1}\right) \check{Z} \check{\check{z}}_{1}, \ldots,\left(\prod_{i=2}^{n-1} N\left(\varrho_{i}, \xi_{i}\right)\right) \check{Z} \check{\check{z}}_{1}\right] \\
= & \tilde{\sigma} \check{Z} L_{n-1}^{\mathrm{rat}}\left(\check{A}, \check{B}, \check{\boldsymbol{z}}_{1}, \Xi, \mathrm{P}\right) .
\end{aligned}
$$

Based on Lemma 3.1 we can now guarantee that the first $n-1$ columns of $\hat{Z}$ are equal to the first $n-1$ columns of $\check{Z}$ multiplied with some $n-1 \times n-1$ unitary block diagonal matrix $D_{\left(s_{1}, \ldots, s_{m}\right)}$. Observe that this also determines $\hat{\boldsymbol{z}}_{n}=\breve{\sigma} \check{\boldsymbol{z}}_{n},|\breve{\sigma}|=1$. This concludes the proof.

In [6, Theorem 6.1] it is shown that an RQZ step with shift $\varrho$ on a Hessenberg pencil with pole tuple $\Xi=\left(\xi_{1}, \ldots, \xi_{n-1}\right)$ and new pole $\xi_{n}$ performs nested subspace iteration accelerated by

$$
q_{k}^{Q}(z)=\frac{z-\varrho}{z-\xi_{k}}, \quad \text { and } \quad q_{k}^{Z}(z)=\frac{z-\varrho}{z-\xi_{k+1}},
$$

for the $k$ th column vector of respectively $Q$ and $Z$. Based on Lemma 2.7, this can be extended to block Hessenberg pencils under the condition that the partition $s$ prior to the multishift, multipole RQZ step is the same as the partition $\hat{s}$ afterwards. We omit this generalization as the condition $s=\hat{s}$ limits the practical application of the theoretical result. Combining [6, Theorem 6.1] with Lemma 2.7, it is clear, however, that in the multishift, multipole RQZ method shifts that have been swapped along the subdiagonal of the block Hessenberg pencil will lead to deflations at the end of the pencil, while poles that have been moved to the front of the pencil lead to convergence of eigenvalues at the beginning. This holds under the assumption that a good choice of poles and shifts is made.

4. Numerical considerations. In this section, we discuss numerical experiments related to the pole introduction and swapping operations and draw conclusions for the practical implementation of the multishift, multipole RQZ method.

4.1. Introducing pole blocks. In finite precision arithmetic, the introduction of a large amount of poles in a block Hessenberg pencil via the computation of the vectors as described in (19) and (23) becomes increasingly inaccurate already for small to medium blocksizes. This comes as no surprise. Kressner [15] studied the use of larger bulges in the QR method and made a connection between the introduction 
of the multishift block in the Hessenberg matrix and the pole placement problem in systems and control theory. It has been shown in control theory that placing many poles in a high dimensional system is intrinsically ill-conditioned [10].

To illustrate the increasing inaccuracy of the pole introduction we have performed a numerical experiment for which the results are summarized in Figure 1. We introduced pole blocks containing $\ell=2,4,6, \ldots, 30$ randomly generated pairs of complexconjugate shifts $\varrho_{i}$ in a real-valued Hessenberg matrix, a real-valued Hessenberg pencil, and a real-valued block Hessenberg pencil with leading blocksize $\ell$. The procedure based on (19) was used for this. All problems are of size $n=100$. The Hessenberg matrix is obtained from the Hessenberg reduction of a randomly generated matrix with normally distributed entries with mean 0 and variance 1 . In this case the shift vector $\boldsymbol{x}$ is computed in the classical way [18] which is compatible with (19). Then an orthonormal matrix $Q$ is computed having $\boldsymbol{q}_{1}=\boldsymbol{x}$. The shifts are introduced as $Q^{T}(A, I)$, which is a block Hessenberg pencil. The actual shifts $\hat{\varrho}_{i}$ are then computed as the eigenvalues of the leading subdiagonal block of $Q^{T}(A, I)$. The black line in Figure 1 shows the median absolute error $\left|\varrho_{i}-\hat{\varrho}_{i}\right|$ over all shifts and 100 repetitions of the experiment. The red line in Figure 1 shows the results of the same experiment but now starting from a Hessenberg pencil $(A, B)$ where each individual matrix is generated as before. Now a procedure based (19) is used to compute $\boldsymbol{x}$. Finally, the blue line shows the results when $(A, B)$ is initially a block Hessenberg pencil with leading blocksize $s_{1}=\ell$ and all other blocks of size 1 .

We remark that, in all three experiments, we obtain a block Hessenberg pencil with partition $(\ell, 1, \ldots, 1)$ after the pole block has been introduced. The only difference is the procedure to compute $\boldsymbol{x}$ and the form of the pencil prior the pole introduction.

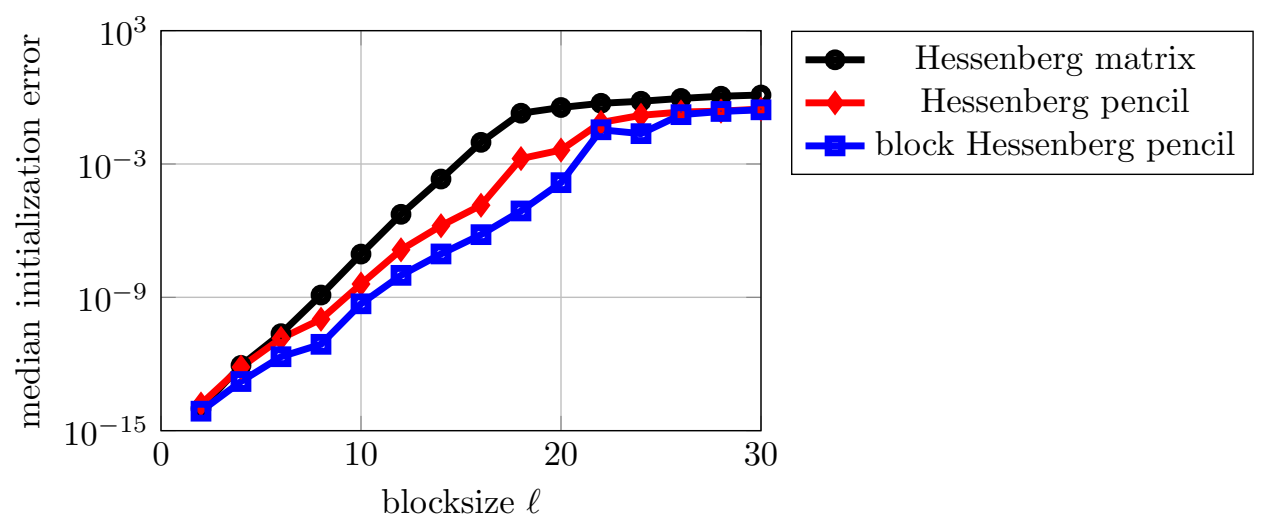

FIG. 1. Initialization error in function of blocksize for multishift $Q R$ (Hessenberg matrix), $R Q Z$ (Hessenberg pencil), and multishift, multipole RQZ (block Hessenberg pencil). Median result over 100 randomly generated problems of size $n=100$.

We observe from Figure 1 that the accuracy of the shifts rapidly decreases for larger blocksizes in all three cases. We conclude from this experiment that the blocksize should be limited in a practical implementation in order to avoid losing all accuracy in the shifts already at the initialization stage. Indeed, there is not much hope for an effective multishift, multipole RQZ sweep if the shifts that are introduced in the block Hessenberg pencil have few to none significant digits in common with the intended shifts. Nonetheless, Watkins [18] showed that in a multishift QR iteration 
shifts that are off at start of the sweep can still come into focus later on.

4.2. Swapping pole blocks. Swapping pole blocks of sizes $s_{i}$ and $s_{i+1}$ requires the solution of a linear system with Kronecker product structure of size $2 s_{i} s_{i+1} \times$ $2 s_{i} s_{i+1}[11,13]$. The computational cost rapidly grows for increasing blocksize.

In case $s_{i}=1$ and $s_{i+1}=1$, or $s_{i}=2$ and $s_{i+1}=1$, the swap can be performed directly based on the computation of an eigenvector [17]. This procedure is norm-wise backward stable.

4.3. Conclusion. In order to limit both the computational cost of the pole introduction and swapping, and the loss of accuracy, we propose a tightly-packed small-block multishift, multipole RQZ sweep. The shifts and poles are tightly-packed similar to [6, Section 4.4].

We represent real poles as subdiagonal blocks of dimension $1 \times 1$ and complexconjugate pairs are represented as subdiagonal blocks of dimension $2 \times 2$ of the form,

$$
\left[\begin{array}{ll}
a_{11} & a_{12} \\
a_{21} & a_{22}
\end{array}\right],\left[\begin{array}{ll}
b_{11} & b_{12} \\
& b_{22}
\end{array}\right]
$$

This standard form has the advantage that $B$ is always an upper Hessenberg matrix throughout the iteration and it simplifies the deflation criterion based on Definition 2.5. The $i$ th pole along the subdiagonal is considered deflated if,

$$
\left|a_{i+1, i}\right|<c \epsilon_{m}\left(\left|a_{i, i}\right|+\left|a_{i+1, i+1}\right|\right), \quad \text { and, } \quad\left|b_{i+1, i}\right|<c \epsilon_{m}\left(\left|b_{i, i}\right|+\left|b_{i+1, i+1}\right|\right),
$$

in the case of a single pole. Here, $\epsilon_{m}$ is the machine precision and $c$ is a small constant. If the $i$ th pole is a double pole in standard form (26), we consider it deflated if either,

$$
\begin{aligned}
\left|a_{i+1, i}\right|+\left|a_{i+2, i}\right| & <c \epsilon_{m}\left(\left|a_{i, i}\right|+\left|a_{i+1, i+1}\right|\right), \quad \text { and, } \\
\left|b_{i+1, i}\right| & <c \epsilon_{m}\left(\left|b_{i, i}\right|+\left|b_{i+1, i+1}\right|\right),
\end{aligned}
$$

or,

$$
\begin{aligned}
\left|a_{i+2, i}\right|+\left|a_{i+2, i+1}\right| & <c \epsilon_{m}\left(\left|a_{i+1, i+1}\right|+\left|a_{i+2, i+2}\right|\right), \quad \text { and, } \\
\left|b_{i+2, i+1}\right| & <c \epsilon_{m}\left(\left|b_{i+1, i+1}\right|+\left|b_{i+2, i+2}\right|\right) .
\end{aligned}
$$

Deflations in the first block column and last block row of the pencil are also checked according to Definition 2.5. The first pole block of size $s_{1}=1$ or 2 can be deflated whenever there exists an $\left(s_{1}+1\right) \times\left(s_{1}+1\right)$ orthogonal matrix $Q$ such that,

$$
Q^{T}\left(\left[\begin{array}{l}
\boldsymbol{a}_{1,1}^{T} \\
A_{2,1}
\end{array}\right],\left[\begin{array}{l}
\boldsymbol{b}_{1,1}^{T} \\
B_{2,1}
\end{array}\right]\right)=\left(\left[\begin{array}{c}
A_{1,1} \\
\mathbf{0}^{T}
\end{array}\right],\left[\begin{array}{c}
B_{1,1} \\
\mathbf{0}^{T}
\end{array}\right]\right)
$$

Here, the last row is considered numerically zero according to a relative tolerance similar to (27)-(29). Again, we make use of the standard form (26) to efficiently check if a suitable deflation transformation $Q$ can be computed in case $s_{1}=2$. A similar approach is used to check for deflations in the last block row.

The swapping transformations are computed according to the procedures described in [17] in case either $s_{i}=1, s_{i+1}=1$, or both of size 1 . If $s_{i}=s_{i+1}=2$, the transformations are computed according to [11, 13]. However, this method is not norm-wise backward stable. It leads to occasional non-negligible off-diagonal blocks when an ill-conditioned $2 \times 2$ block close to convergence is involved. In this case, the transformation is refined [5] to decrease the norm of the off-diagonal block. Our numerical experiments indicated that iterative refinement is required in about $5 \%$ of all $2 \times 2$ with $2 \times 2$ swaps during a typical RQZ iteration.

In Section 6, we will describe our final multishift, multipole RQZ algorithm and test it in a series of numerical experiments. 
5. Aggressive early deflation. Aggressive early deflation (AED) significantly speeds up the convergence of the QR [4] and QZ [12] methods by identifying deflatable eigenvalues before classical deflation criteria are able to detect them. This avoids the reuse of converged shifts in subsequent iterations, thereby initiating convergence of other eigenvalues sooner.

In this section, we describe how aggressive early deflation is implemented for the RQZ method. The process exists out of 3 stages and is summarized in Figure 2. Because the shifts lead to convergence in the bottom-right corner of the pencil and the poles cause convergence in the upper-left corner, AED can be performed at both sides of the pencil. We present the description of the AED process simultaneously for the upper-left and bottom-right sides of the pencil, but they can be treated separately in a practical implementation. The deflation window sizes are $w_{e}$ for the bottom-right side and $w_{s}$ for the upper-left side of the pencil. The window sizes are chosen such that they cover an integer number of blocks, avoiding thereby subdivision of $2 \times 2$ blocks. The deflation windows are shown in Pane I of Figure 2.

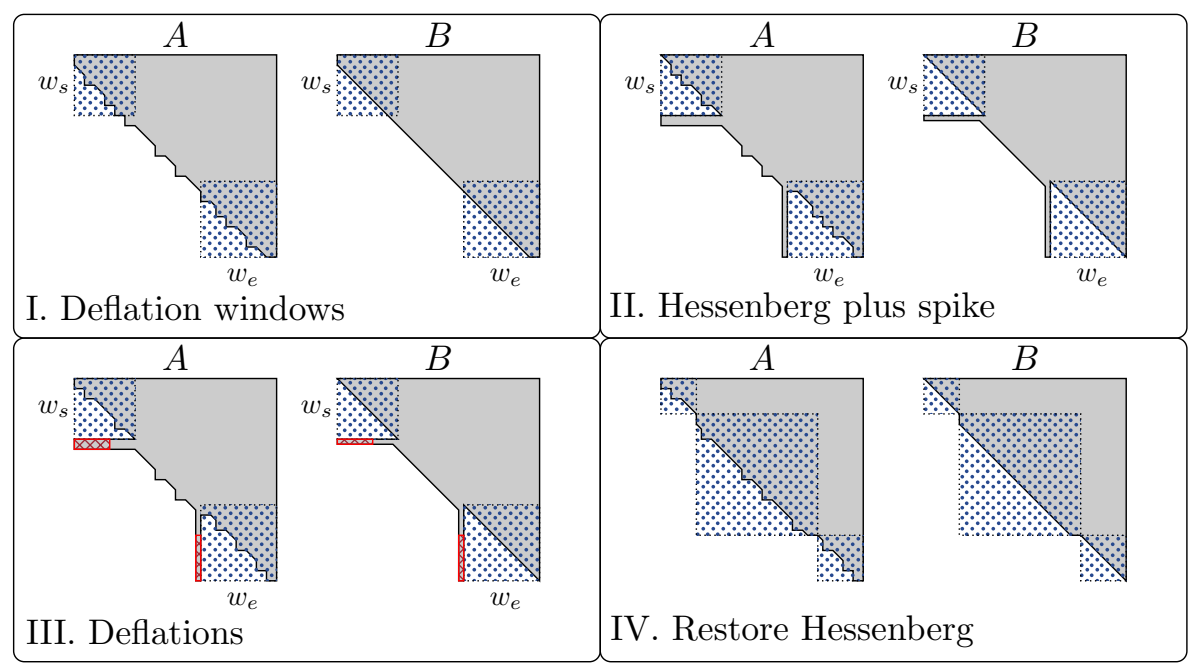

FIG. 2. Visualization of the three stages of aggressive early deflation for block Hessenberg pencils; both at the front and back of the pencil. The matrix $A$ is in block Hessenberg form with $2 \times 2$ blocks representing complex conjugate pairs of shifts, the matrix $B$ is in Hessenberg form.

In the first phase, shown in pane II of Figure 2, the parts of the pencil within the deflation windows are reduced to real Schur form. This can be done with the RQZ method as all subpencils in the deflation windows are in block Hessenberg form. The pencil $(A, B)$ is subdivided as,

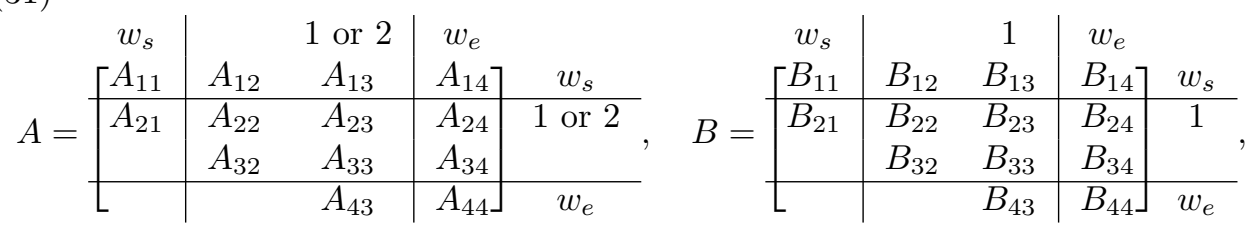

and the subpencils $\left(A_{11}, B_{11}\right)$ and $\left(A_{44}, B_{44}\right)$ are the upper-left and bottom-right 
deflation windows. Their reduction to real Schur form is given by,

$$
\left(T_{11}, S_{11}\right)=Q_{s}^{T}\left(A_{11}, B_{11}\right) Z_{s}, \quad \text { and }, \quad\left(T_{44}, S_{44}\right)=Q_{e}^{T}\left(A_{44}, B_{44}\right) Z_{e},
$$

which, when applied as an equivalence transformation to $(A, B)$ gives the following result:

$\check{A}=\left[\begin{array}{c|cc|c}T_{11} & Q_{s}^{T} A_{12} & Q_{s}^{T} A_{13} & Q_{s}^{T} A_{14} Z_{e} \\ \hline A_{21} Z_{s} & A_{22} & A_{23} & A_{24} Z_{e} \\ & A_{32} & A_{33} & A_{34} Z_{e}\end{array}\right], \check{B}=\left[\begin{array}{c|cc|c}S_{11} & Q_{s}^{T} B_{12} & Q_{s}^{T} B_{13} & Q_{s}^{T} B_{14} Z_{e} \\ \hline B_{21} Z_{s} & B_{22} & B_{23} & B_{24} Z_{e} \\ & Q_{32}^{T} & B_{33} & B_{34} Z_{e} \\ \hline & & Q_{e}^{T} A_{43} & T_{44}\end{array}\right]$.

The blocks $\left(A_{21}, B_{21}\right) Z_{s}$ and $Q_{e}^{T}\left(A_{43}, B_{43}\right)$ are the spikes shown in pane II of Figure 2. Because $B$ is an upper Hessenberg matrix by (26), $B_{21}=b_{w_{s}+1, w_{s}} \boldsymbol{e}_{w_{s}}^{T}$ is of dimension $1 \times w_{s}$ and $B_{43}=b_{n-w_{e}+1, n-w_{e}} \boldsymbol{e}_{1}$ is of dimension $w_{e} \times 1$. The spikes at the side of $A$ can be of dimension $2 \times w_{s}$ or $w_{e} \times 2$ if there is a $2 \times 2$ block just after the deflation window in the upper-left side of the pencil (the example of Figure 2 illustrates this situation), or right before the deflation window at the bottom-right side of the pencil. In this case, the 2 rows of $A_{21} Z_{s}$ are scalar multiples of each other. The same holds for the 2 columns of $Q_{e}^{T} A_{43}$. We denote with $\boldsymbol{p}_{s}^{B}=b_{w_{s}+1, w_{s}} \boldsymbol{e}_{w_{s}}^{T} Z_{s}$ the spike at the upperleft deflation window of $B$. Similarly, $\boldsymbol{p}_{s}^{A}=\zeta \boldsymbol{e}_{w_{s}}^{T} Z_{s}$, with $\zeta$ equal to the maximum of $\left|a_{w_{s}+1, w_{s}}\right|$ and $\left|a_{w_{s}+2, w_{s}}\right|$, denotes the spike at the upper-left side of $A$

The second phase in the AED process is illustrated in Pane III of Figure 2 and entails testing for deflatable eigenvalues inside the deflation windows. The deflation test starts at the left of the spikes $\boldsymbol{p}_{s}^{A}$ and $\boldsymbol{p}_{s}^{B}$. If there is a $1 \times 1$ real eigenvalue located at this position, we test if:

$$
\left|\boldsymbol{p}_{s, 1}^{A}\right|<c \epsilon_{m}\left(\left|a_{1,1}\right|+\left|a_{2,2}\right|\right), \quad \text { and, } \quad\left|\boldsymbol{p}_{s, 1}^{B}\right|<c \epsilon_{m}\left(\left|b_{1,1}\right|+\left|b_{2,2}\right|\right) .
$$

If there is a $2 \times 2$ complex conjugate pair of eigenvalues at this position, we test if:

$$
\left|\boldsymbol{p}_{s, 1}^{A}\right|+\left|\boldsymbol{p}_{s, 2}^{A}\right|<c \epsilon_{m}\left\|A_{1: 2,1: 2}\right\|_{F}, \quad \text { and, } \quad\left|\boldsymbol{p}_{s, 1}^{B}\right|+\left|\boldsymbol{p}_{s, 2}^{B}\right|<c \epsilon_{m}\left\|B_{1: 2,1: 2}\right\|_{F} .
$$

If the first eigenvalue is deflatable according to (34) or (35), the corresponding spike elements in $\boldsymbol{p}_{s}^{A}$ and $\boldsymbol{p}_{s}^{B}$ are set to zero and the next eigenvalue is tested according to the same criterion. If the first eigenvalue is not deflatable, another eigenvalue that has not yet been tested, is swapped to the front of the spike. Then it is checked if this is deflatable according to (34) or (35). This procedure is continued until all deflatable eigenvalues inside the deflation window are identified. The swapping of eigenvalues within the deflation window does not change the form of (32) but the equivalences $\hat{Q}_{s}$ and $\hat{Z}_{s}$ are changed which also changes $\hat{\boldsymbol{p}}_{s}$ and $\left(\hat{T}_{11}, \hat{S}_{11}\right)$. The same strategy is used for AED at the bottom-right side of the pencil. In pane III of Figure 2 all spike elements that have been zeroed are marked in red.

In the third and last phase, the nonzero spike elements are handled in such a way that the (block) Hessenberg form is restored. The restored form is shown in pane IV of Figure 2, where the larger block in the middle is in block Hessenberg form and the smaller blocks at the upper-left and bottom-right side of the pencil are in real Schur form. The block Hessenberg restoration is achieved by a sequence of rotations as follows. Assume that the spikes after the deflation procedure of second phase are $\hat{\boldsymbol{p}}_{s}=\hat{\zeta} \boldsymbol{e}_{w_{s}}^{T} \hat{Z}_{s}$ and that the first $i$ entries in $\hat{\boldsymbol{p}}_{s}$ are zeroed during the deflation step. We then compute rotations $G_{i+1}, \ldots, G_{w_{s}-1}$ such that,

$$
\hat{\boldsymbol{p}}_{s} G_{i+1}, \ldots, G_{w_{s}-1}=\hat{\zeta} \boldsymbol{e}_{w_{s}}^{T} \hat{Z}_{s} G_{i+1}, \ldots, G_{w_{s}-1}=\sigma \hat{\zeta} \boldsymbol{e}_{w_{s}}^{T} .
$$


Updating $\tilde{Z}_{s}=\hat{Z}_{s} G_{i+1}, \ldots, G_{w_{s}-1}$ gives the final equivalence such that the block Hessenberg form is restored. The same idea is used for the deflation window at the bottom-right side of the pencil. We remark that for complex-valued problems the Hessenberg form can be restored in the third phase by a row or column permutation for respectively AED at the upper-left or bottom-right side of the pencil.

6. Numerics. The numerical tests have been performed on an Intel Xeon E5$2697 \mathrm{v} 3 \mathrm{CPU}$ with 14 cores and 128GB of RAM. Our implementation of the multishift, multipole RQZ method with aggressive deflation is compiled with gfortran version 4.8.5 using compilation flag -03. LAPACK [1] and BLAS are referenced via -llapack and -lblas. The library libRQZ supports both real-valued (dRQZm) and complexvalued (zRQZm) problems.

6.1. dRQZm and zRQZm. As discussed in Section 4, dRQZm uses $1 \times 1$ blocks for real poles and $2 \times 2$ blocks for pairs of complex-conjugate shifts, zRQZm always uses $1 \times 1$ blocks. Both algorithms proceed as follows:

I. Check for deflations at the upper-left side of the pencil using AED with window size $w_{s}$.

II. Check for interior deflations along the subdiagonal.

III. Compute $m$ shifts as the eigenvalues of the trailing $m \times m$ block with the RQZ method and introduce these as consecutive poles in the first $m$ subdiagonal positions of the block Hessenberg pencil. This is achieved by using the operations of Subsection 2.3. The involved transformations are accumulated and the pencil is updated by level 3 BLAS matrix-matrix multiplication.

IV. Chase the batch of $m$ shifts to the last $m$ positions on the subdiagonal of the block Hessenberg pencil. The chasing is performed by repeatedly swapping the $m$ shifts with the next $k$ poles. Every time one sequence of swaps is computed, all transformations are accumulated and the pencil is updated by level 3 BLAS matrix-matrix multiplication.

V. Check for deflations at the bottom-right side of the pencil using AED with window size $w_{e}$.

VI. Compute $m$ poles as the eigenvalues of the leading $m \times m$ block with the RQZ method and introduce these as consecutive poles in the last $m$ subdiagonal positions of the block Hessenberg pencil. This is achieved by using the operations of Subsection 2.3. The involved transformations are accumulated and the pencil is updated by level 3 BLAS matrix-matrix multiplication.

This algorithm actively chases shifts from the upper-left corner to the bottom-right corner. This typically leads to fast convergence of eigenvalues near the bottom-right side of the pencil. The swapping also slowly moves the poles that are introduced at the bottom-right corner to the upper-left side of the pencil which, in turn, induces convergence of eigenvalues near the upper-left corner of the pencil.

The settings used in libRQZ are summarized in Table 1. The first column lists the size of the pencil. The second column lists the batch size $m$ of shifts that are handled in one iteration. The third column lists the swap size $k$ : after the $m$ shifts have been swapped with the next $k$ poles, the transformations are accumulated and the entire pencil is updated with a BLAS call. In our experience, choosing $k$ equal to $m$ gives the best performance. The fourth column lists the window size $w_{e}$ for aggressive early deflation at the bottom-right side of the pencil. Finally, the fifth column lists the window size $w_{s}$ for aggressive early deflation at the upper-left side of the pencil.

We compare zRQZm and dRQZm with respectively ZHGEQZ and DHGEQZ from LAPACK [1] in terms of speed and accuracy. 
TABLE 1

libRQZ settings: $n$ problem size, $m$ step multiplicity, $k$ swap range, $w_{e}$ AED window size at the bottom-right side of the pencil, $w_{s}$ AED window size at the upper-left side of the pencil.

\begin{tabular}{l|c|c|c|c}
$n$ & $m$ & $k$ & $w_{e}$ & $w_{s}$ \\
\hline$[1 ; 80[$ & $1-2$ & $1-2$ & $1-2$ & $1-2$ \\
{$[80 ; 150[$} & 4 & 4 & 6 & 4 \\
{$[150 ; 250[$} & 8 & 8 & 10 & 4 \\
{$[250 ; 501[$} & 16 & 16 & 18 & 6 \\
{$[501 ; 1001[$} & 32 & 32 & 34 & 10 \\
{$[1001 ; 3000[$} & 64 & 64 & 66 & 16 \\
{$[3000 ; 6000[$} & 128 & 128 & 130 & 32 \\
{$[6000 ; \infty[$} & 256 & 256 & 266 & 48
\end{tabular}

6.2. Random problems. For our first numerical experiment, we have generated random matrix pairs of increasing dimension. The entries of the matrices are drawn from the standard normal distribution. The experiment is performed both for realvalued and complex-valued matrix pairs; for the latter class of problems, both the real and imaginary part are randomly generated.

The matrix pairs are initially reduced to Hessenberg, triangular form by means of the LAPACK [1] routines XGEQRFP and xGGHRD. After this initial reduction, the matrix pairs are further reduced to (real) generalized Schur form, $(S, T)=Q^{*}(A, B) Z$, with libRQZ and LAPACK [1]. In all cases, the entire Schur decomposition is computed.
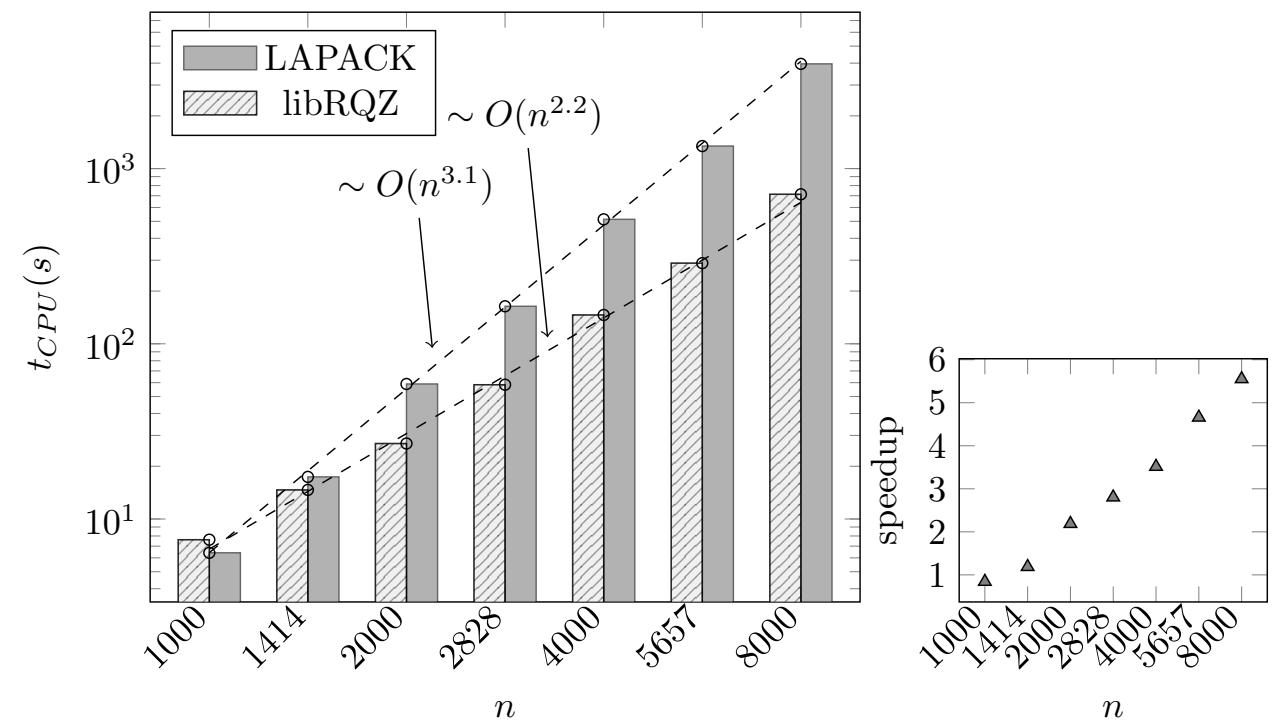

FIG. 3. CPU time of DHGEQZ of LAPACK and dRQZm of libRQZ on randomly generated realvalued matrix pencils (left). Speedup of libRQZ over LAPACK (right).

The left part of Figure 3 shows the CPU time of dRQZm and DHGEQZ for problems of size 1000 up to 8000 on a loglog scale. The dashed lines indicate the slopes of the time complexity in function of problem size, which are estimated in a least-squares sense. The least-square fits are computed based on the $\left(n_{i}, t_{i}\right)$ data indicated with the circular 
markers that show the exact height of the bars in the graph. For DHGEQZ we observe an empirical time complexity close to $O\left(n^{3}\right)$, while the empirical time complexity of dRQZm is significantly lower than $O\left(n^{3}\right)$ with a leading exponent close to 2.2. This improved time complexity can be attributed to the effectiveness of aggressive early deflation in combination with the rational iteration leading to occasional deflations situated more in the interior part of the pencil.

The right part of Figure 3 shows the speedup achieved by dRQZm over DHGEQZ. The crossover point where dRQZm becomes faster than DHGEQZ is situated between $n=1000$ and $n=1414$. Our method, dRQZm, is slower than DHGEQZ for problems of smaller size because the computational overhead of computing swaps of $2 \times 2$ with $2 \times 2$ blocks and $2 \times 2$ with $1 \times 1$ blocks leads to larger lower-order terms in the time complexity.
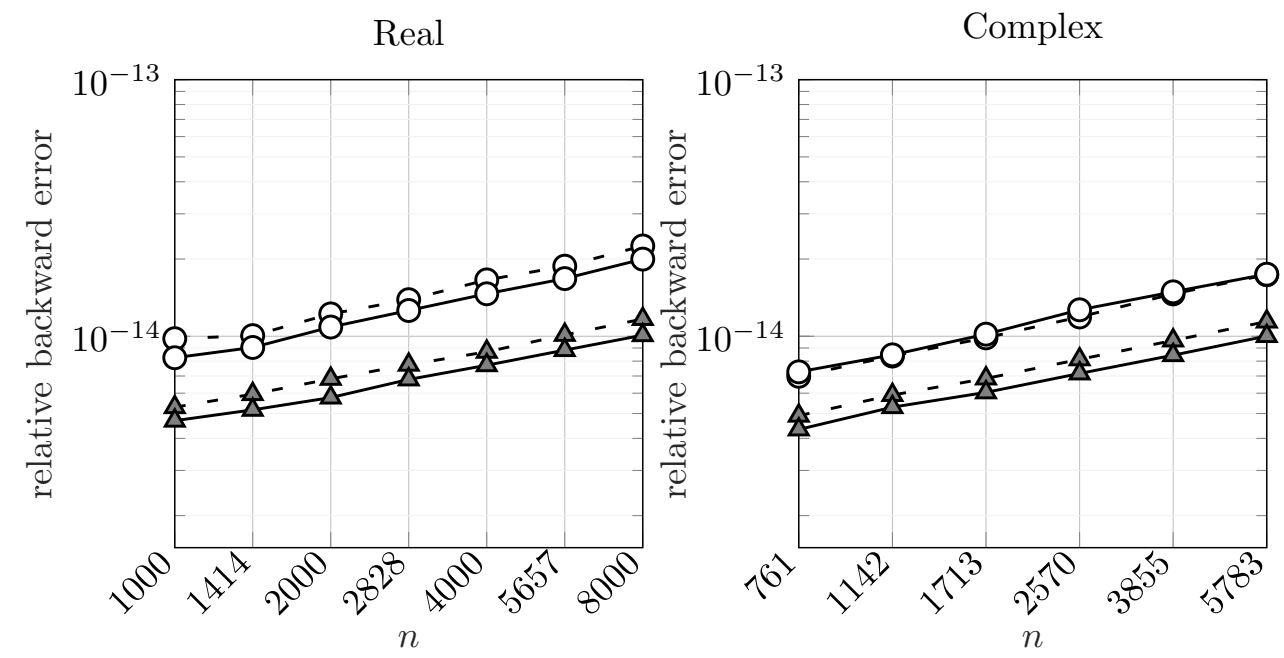

FIG. 4. Relative backward error on Schur decomposition computed with LAPACK (circles) and libRQZ (triangles) on $A$ (full lines) and $B$ (dashed lines). Both for real-valued (left) and complex-valued (right) randomly generated matrix pairs.

The left part of Figure 4 shows the relative backward errors,

$$
\left\|T-Q^{T} A Z\right\|_{F} /\|A\|_{F}, \quad \text { and, } \quad\left\|S-Q^{T} B Z\right\|_{F} /\|B\|_{F},
$$

on the generalized real Schur decompositions obtained with dRQZm and DHGEQZ. We observe that the relative backward errors of dRQZm are about half of these of DHGEQZ.

Figure 5 shows the results of an experiment similar to Figure 3 but for complexvalued pencils. Again, ZHGEQZ shows an empirical time complexity larger than $O\left(n^{3}\right)$, while zRQZm stays below $O\left(n^{3}\right)$. The crossover point where ZHGEQZ is faster than zRQZm is not shown in Figure 5 , but is situated around $n=200$. This is significantly lower than for $\mathrm{dRQZm}$ and is explained by the fact that only $1 \times 1$ with $1 \times 1$ swaps are used in this case. These have a lower computational overhead than larger swaps. The right part of Figure 4 shows the relative backward errors on the generalized Schur decompositions for the complex-valued pencils. Again, the relative backward error of zRQZm is about half of ZHGEQZ. 

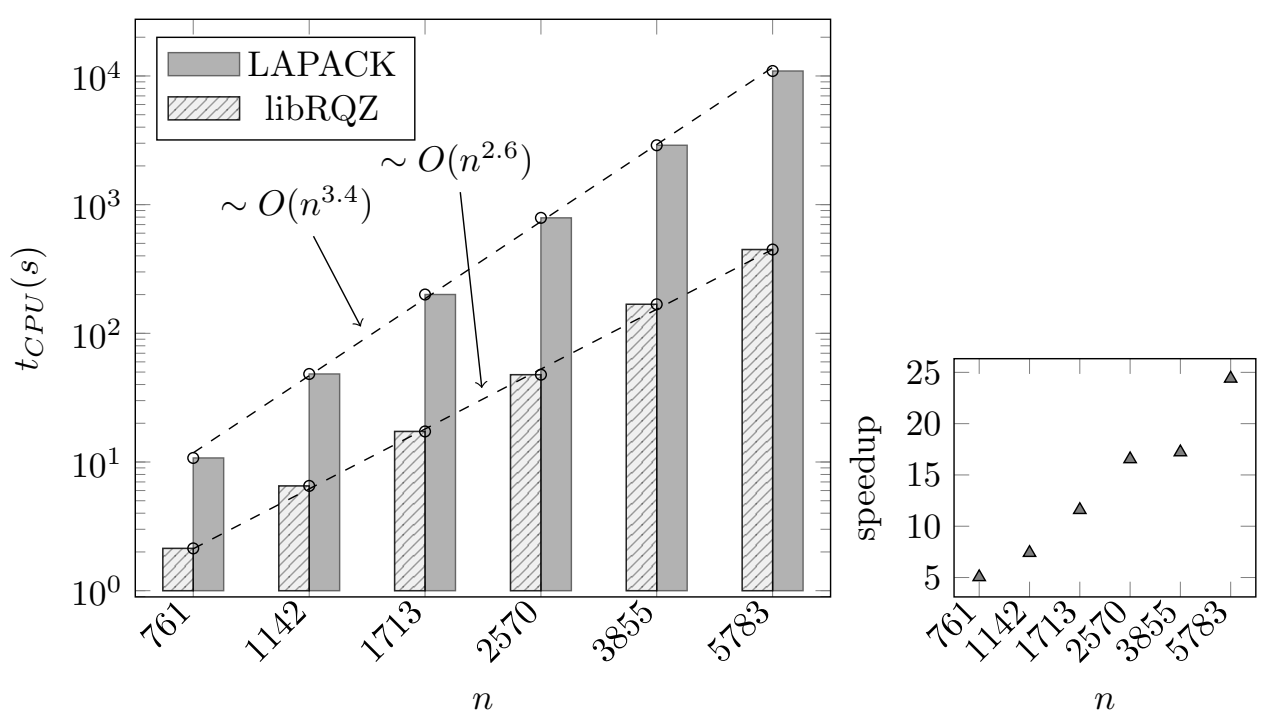

FIG. 5. CPU time of ZHGEQZ of LAPACK and $z R Q Z m$ of libRQZ on randomly generated complexvalued matrix pencils (left). Speedup of libRQZ over LAPACK (right).

6.3. Problems from applications. In this section we test libRQZ on five pencils originating from applications. We study the cavity and obstacle flow pencils generated with IFISS $[7,8]$. The same pencils were studied in our initial paper on the RQZ method [6]. Besides these pencils, we have selected two pencils from Matrix market [2] originating from the MHD collection and the rail pencil from the Oberwolfach benchmark collection [14].

The results of the numerical tests are summarized in Table 2. The table lists the CPU time and maximum of the relative backward errors on $A$ and $B$ for the generalized real Schur form computed with LAPACK [1] and libRQZ. Again, libRQZ requires less CPU time and has the smaller backward error.

TABLE 2

$C P U$ times and maximum relative backward error on the generalized real Schur form computed with LAPACK and libRQZ for pencils originating from applications.

\begin{tabular}{l|c|c|c|c|c} 
& & \multicolumn{2}{|c|}{ DHGEQZ } & \multicolumn{2}{c}{ dRQZm } \\
Problem & $n$ & $t_{\mathrm{CPU}}(s)$ & max error $(A, B)$ & $t_{\mathrm{CPU}}(s)$ & max error $(A, B)$ \\
\hline Cavity Flow & 2467 & 50.1 & $1.2 \cdot 10^{-14}$ & 20.4 & $4.7 \cdot 10^{-15}$ \\
Obstacle Flow & 2488 & 64.0 & $9.9 \cdot 10^{-15}$ & 27.9 & $5.9 \cdot 10^{-15}$ \\
MHD3200 & 3200 & 60.8 & $9.0 \cdot 10^{-15}$ & 39.6 & $3.1 \cdot 10^{-15}$ \\
MHD4800 & 4800 & 194.1 & $1.4 \cdot 10^{-14}$ & 92.2 & $3.4 \cdot 10^{-15}$ \\
RAIL & 5177 & 1287.5 & $1.7 \cdot 10^{-13}$ & 87.5 & $1.1 \cdot 10^{-14}$
\end{tabular}

7. Conclusion and future work. In this paper we have generalized the rational QZ method from Hessenberg to block Hessenberg pencils. This allows for the use of complex conjugate shifts and poles in real arithmetic. Numerical considerations have shown that medium to large multiplicities are unfavorable due to inherent inaccuracies and an increasing computational complexity. In the spirit of recent developments for the QR [3, 4] and QZ [12] methods, this urged us to use small shift 
and pole multiplicities, but they can be tightly-packed together. This approach maintains accurate shifts and poles in combination with level 3 BLAS performance. We also implemented the aggressive early deflation strategy for block Hessenberg pencils. Numerical experiments indicated that this combination leads to an efficient algorithm for the generalized eigenvalue problem that can outperform LAPACK [1] in terms of speed, accuracy, and time complexity.

In a future update of libRQZ, we plan to include the option to use bidirectional RQZ sweeps that actively chase poles from the bottom-right of the pencil to the upperleft side in parallel to chasing shifts from the upper-left to the bottom-right side of the pencil. Bidirectional chasing can, for a large part, be performed independently in both directions. It is hence an excellent opportunity for shared-memory parallelization. On the theoretical side, a further investigation of shift and pole selection strategies that stimulate interior deflations would be an interesting undertaking.

Acknowledgements. The authors are grateful to Paul Van Dooren and Nicola Mastronardi for their help with the iterative refinement procedure for $2 \times 2$ with $2 \times 2$ swaps [5] which was essential for handling $2 \times 2$ blocks accurately.

\section{REFERENCES}

[1] E. Anderson, Z. Bai, C. Bischof, S. Blackford, J. Demmel, J. Dongarra, J. Du Croz, A. Greenbaum, S. Hammarling, A. McKenney, and D. Sorensen, LAPACK Users' Guide, Society for Industrial and Applied Mathematics, Philadelphia, PA, third ed., 1999.

[2] R. F. Boisvert, R. Pozo, K. Remington, R. F. Barrett, and J. J. Dongarra, Matrix market: A web resource for test matrix collections, in Proceedings of the IFIP TC2/WG2.5 Working Conference on Quality of Numerical Software: Assessment and Enhancement, London, UK, 1997, Chapman \& Hall, Ltd., pp. 125-137.

[3] K. Braman, R. Byers, and R. Mathias, The multishift QR algorithm. Part I: maintaining well-focused shifts and level 3 performance, SIAM J. Matrix Anal. Appl., 23 (2002), pp. 929-947.

[4] K. Braman, R. Byers, and R. Mathias, The multishift QR algorithm. Part II: aggressive early deflation, SIAM J. Matrix Anal. Appl., 23 (2002), pp. 948-973.

[5] D. Camps, N. Mastronardi, R. Vandebril, and P. Van Dooren, Swapping $2 \times 2$ blocks in the Schur and generalized Schur form, (2019). Submitted.

[6] D. Camps, K. Meerbergen, And R. Vandebril, A rational QZ method, (2018), https://arxiv. org/abs/1802.04094. Submitted.

[7] H. Elman, A. Ramage, and D. Silvester, Algorithm 866: IFISS, a Matlab toolbox for modelling incompressible flow, ACM Trans. Math. Softw., 33 (2007), pp. 2-14.

[8] H. Elman, A. Ramage, And D. Silvester, IFISS: A computational laboratory for investigating incompressible flow problems, SIAM Rev., 56 (2014), pp. 261-273.

[9] J. G. F. Francis, The QR Transformation-Part 2, Comput. J., 4 (1962), pp. 332-345, https: //doi.org/10.1093/comjnl/4.4.332.

[10] C. He, A. J. Laub, and V. Mehrmann, Placing plenty of poles is pretty preposterous, in Preprint SPC 95-17, Forschergruppe Scientific Parallel Computing, Fak. f. Mathematik, TU Chemnitz-Zwickau, May 1995.

[11] B. KÅgStröm, A Direct Method for Reordering Eigenvalues in the Generalized Real Schur form of a Regular Matrix Pair (A, B), Springer Netherlands, Dordrecht, 1993, pp. 195218, https://doi.org/10.1007/978-94-015-8196-7_11.

[12] B. KÅGSTRÖM AND D. KRessner, Multishift variants of the $Q Z$ algorithm with aggressive early deflation, SIAM J. Matrix Anal. Appl., 29 (2007), pp. 199-227.

[13] B. KÅGström ANd P. PoromaA, Computing eigenspaces with specified eigenvalues of a regular matrix pair $(A, B)$ and condition estimation: theory, algorithms and software, Numer. Algorithms, 12 (1996), pp. 369-407.

[14] J. G. Korvink and E. B. Rudnyi, Oberwolfach benchmark collection, in Dimension Reduction of Large-Scale Systems, P. Benner, D. C. Sorensen, and V. Mehrmann, eds., Berlin, Heidelberg, 2005, Springer Berlin Heidelberg, pp. 311-315.

[15] D. Kressner, On the use of larger bulges in the QR algorithm, Electron. Trans. Numer. Anal., 20 (2005), pp. 50-63. 
[16] C. B. Moler and G. W. Stewart, An algorithm for generalized matrix eigenvalue problems, SIAM J. Numer. Anal., 10 (1973), pp. 1-52.

[17] P. VAn Dooren, A generalized eigenvalue approach for solving Riccati equations, SIAM J. Sci. Stat. Comput., 2 (1981), pp. 121-135, https://doi.org/10.1137/0902010.

[18] D. S. WatKIns, The transmission of shifts and shift blurring in the $Q R$ algorithm, Linear Algebra Appl., 241-243 (1996), pp. 877-896. 